MODELING, IDENTIFICATION AND CONTROL, 1996, vOL. 17, NO. 4, 231-259

doi:10.4173/mic.1996.4.1

\title{
Identification of Vibration Modes in a Spacecraft using Nonparametric and Parametric Methods
}

\begin{abstract}
ÅGE SKULLESTAD*
Keywords: Parameter identification, spectral estimation, linear systems, spacecraft

This article describes identification of vibration frequencies in a spacecraft. A mathematical model of a simple spacecraft is built in MATLAG code, and after a theoretical overview, this spacecraft model is simulated, using a torque excitation, to generate time series measurements. These measurements are subsequently used in the identification experiments. Parametric methods, here represented by AR, ARX, ARMA and ARMAX, are the main concern, but nonparametric methods are also discussed.
\end{abstract}

\section{Introduction}

Future utilization of space is expected to require spacecraft consisting of large structures. An increasing need for better pointing accuracy of antennas connected to the space structures puts strong demands on the attitude control systems. Communication antennas already have to be pointed to within 0.03 degree RMS (Joshi 1989). A high precision attitude control system does not only depend on the pointing accuracy of the spacecraft body, but also on the displacement, due to the vibration modes of the flexible structures. Damping/controlling the vibration modes with significant energy will be necessary in high-precision attitude-control systems. Because of the light weight of the large structures, vibration modes will tend to have low damping and low vibration frequencies (natural frequencies) that will be closely spaced and are expected to vary as much as $20-30 \%$, due to splashing and consumption of rocket fuel. Some of the vibration frequencies can also be expected to be lower than the bandwidths of some of the control loops. Knowledge of the vibration frequencies is vital for the design of high precision control systems, as is the need for identifying the vibration frequencies in space.

This paper presents some identification methods that may be used to identify the vibration frequencies in space. Measurements to be used in the identification process are taken from a mathematical model of a spacecraft. The mathematical model consists of a flexible Euler-Bernoulli beam connected to a rigid satellite core. The flexible beam is forced to vibrate and a sensor located somewhere along the beam measures the acceleration of the beam displacement.

This paper first considers nonparametric methods, which can be based on Fourier analysis. Parametric methods are represented in this paper by the AR, ARX, ARMA, ARMAX methods. Parametric methods were originally developed as batch methods, but most batch methods now have recursive counterparts which also will be looked at.

Received 14 September 1995.

*Høysholen i Bieskerud, Postboks 231, 3601 Kongsberg. 


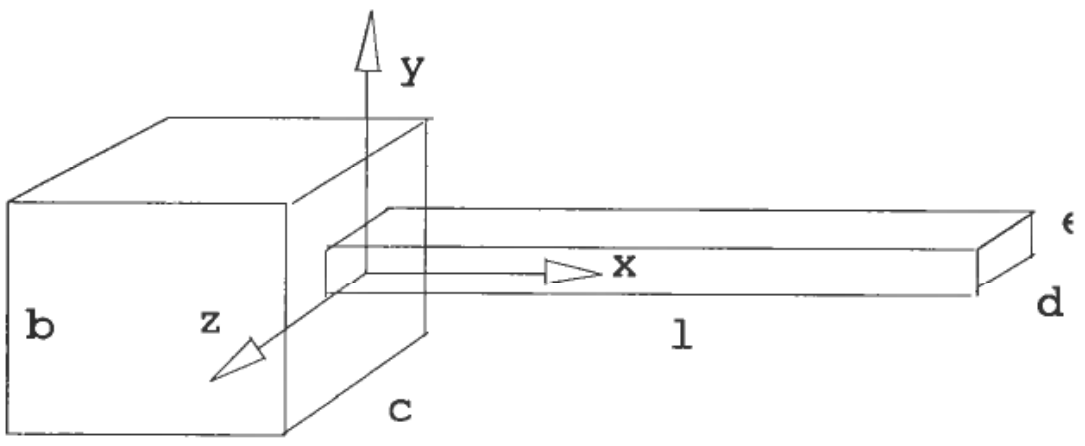

a

Figure 1. Space structure model.

Section 2 presents the mathematical model of the spacecraft. Section 3 describes identification of vibration frequencies using nonparametric methods. Section 4 describes identification of vibration parameters using parametric methods. The properties of the nonparametric and parametric methods are demonstrated by simulations in section 5. Conclusions are found in section 6 .

\section{Mathematical Model of the Spacecraft}

The spacecraft consists of a flexible beam connected to a satellite body, see Figure 1. The flexible beam is modelled as an Euler-Bernoulli beam and the satellite body as a rigid body. The reason for this is that the satellite body has a very stiff construction whereas the beam has a light construction and is highly flexible.

\section{Mathematical Model of an Euler-Bernoulli Beam}

The displacement-time function of an Euler-Bernoulli beam, connected to the large rigid body and undergoing vibrations, is described by the differential equation (Rao S. 1990)

$$
E J \frac{\partial^{4} y(x, t)}{\partial x^{4}}+\rho \frac{\partial^{2} y(x, t)}{\partial t^{2}}=f(x, t)
$$

where $\rho$ is mass/unit length, E is Young's modulus, $J$ is the moment of inertia and $f(x, t)$ is an external force per unit length of the beam. (1) is separable and has the solution

$$
y(x, t)=\sum_{n=1}^{N} W_{n}(x) q_{n}(t)
$$

The displacement of the beam $y(x, t)$ is a function of time and the distance $x$ from the fixed end (connection satellite core/flexible beam). $\mathrm{N}$ is the total number of vibration modes, $q_{n}(t)$ is the time dependent vibration of mode $n$ and $W_{n}(x)$ is the mode shape function of the $\mathrm{n}^{\text {th }}$ vibration mode. $\mathrm{W}_{\mathrm{n}}(\mathrm{x})$ may be found from ordinary textbooks on mechanical engineering, for instance Rao (1990). Although a flexible beam has an infinite number of vibration frequencies, the number of frequencies is in this analysis limited to four, given in Table 1.

Figure 2 shows the mode-shapes of the vibration modes of the flexible beam against beam length.

A spacecraft normally consists of one or several flexible structures connected to a rigid core. Flexible structures connected to the same rigid core often have common 


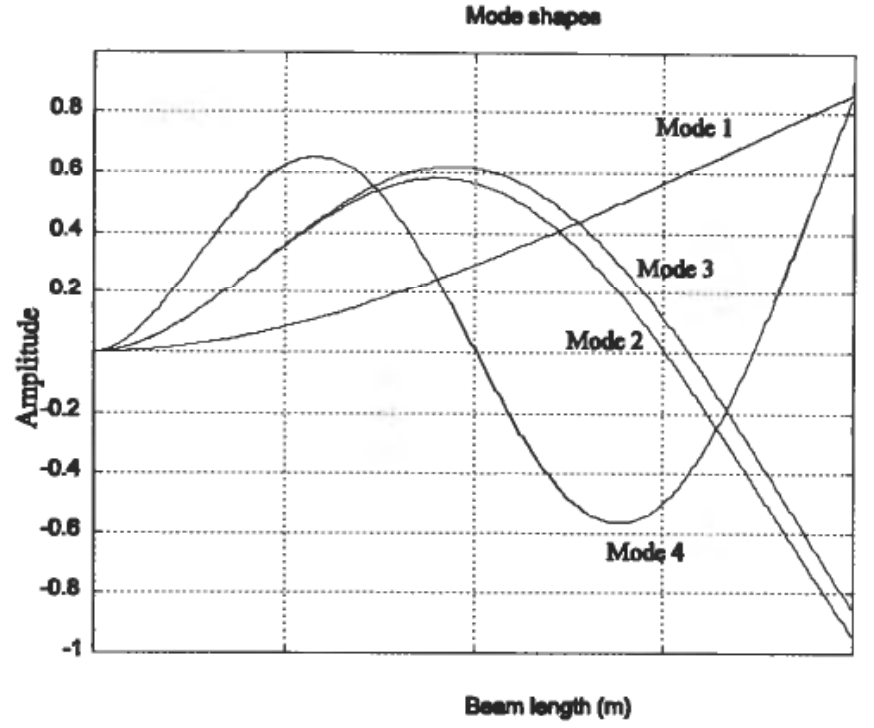

Figure 2. Mode shapes of the vibrations.

Table 1. Frequencies of beam vibrations

\begin{tabular}{lcccc}
\hline & \multicolumn{4}{c}{ Frequencies of the beam vibration } \\
\cline { 2 - 5 } Modes & 1 & 2 & 3 & 4 \\
\hline Frequencies $(\mathrm{Hz})$ & 0.51 & 3.22 & 3.50 & 9.02 \\
\hline
\end{tabular}

frequencies. In order to separate those vibrations which may differ in amplitude, frequency identification is performed separately for each flexible structure, i.e. one or more sensors are located on each flexible structure. One flexible space structure is therefore sufficient to obtain a realistic identification case. The chosen flexible beam may represent a flexible space structure with a small modification. The vibration frequencies of a simple flexible beam are widely spaced, whereas a space structure normally has two or more closely spaced frequencies. The mode shape and frequency of mode 3 in the beam model is modified such that mode 2 and 3 become closely spaced, see Table 1 and Figure 2.

The performance of the attitude control system (i.e. the pointing accuracy) will depend on how many of the vibration modes, with significant energy, that are damped/controlled. Four vibration frequencies, as used in this paper, are expected to

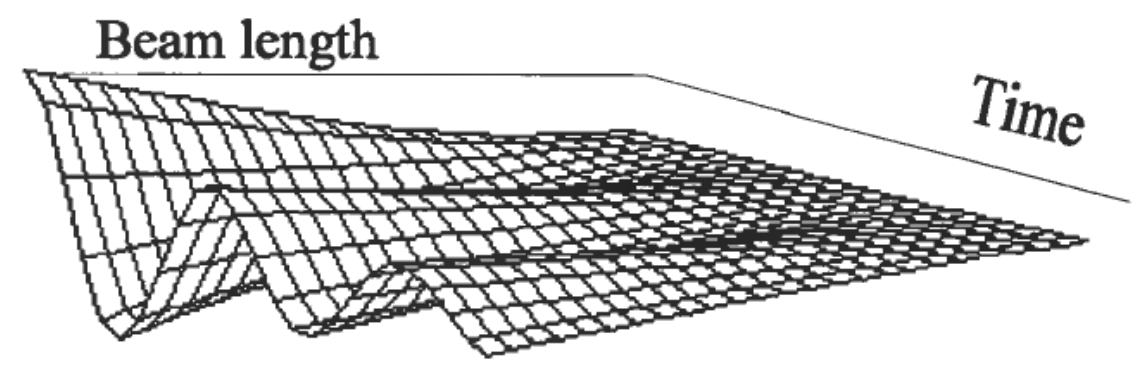

Figure 3. Time-space plot of vibration mode 1. 
give a simple, but realistic model.

Figure 3 shows the time-space plot of the beam vibration due to mode 1 . The damping, which normally is in the order of 0.001 , has been increased to 0.1 in order to aid visualisation.

\section{Mathematical Model of a Rigid Body}

The flexible beam is fixed to the satellite core, which is considered to be rigid. Assuming for the moment the flexible beam to be rigid, the equations of motion referred to a body fixed frame of reference ( $x, y$ and $\mathrm{z}$ in Figure 1) consist of the moment and translation equations shown in (3).

$$
\begin{aligned}
\left(J_{B}+J_{E}\right) \dot{\omega}+m_{B} r_{0} \times a_{0}+m_{E} r_{E} \times a_{0} & =T_{B}+T_{E} \\
\left(m_{B}+m_{E}\right) a_{0}-m_{B} r_{0} \times \dot{\omega}-m_{E} r_{E} \times \dot{\omega} & =F_{B}+F_{E}
\end{aligned}
$$

where

$$
r_{0}=\left[\begin{array}{lll}
\div \frac{a}{2} & 0 & 0
\end{array}\right]^{T}, \quad r_{E}=\left[\begin{array}{lll}
\frac{l}{2} & 0 & 0
\end{array}\right]^{T}
$$

$J_{B}$ and $J_{E}$ are the moments of inertia of the satellite body and the beam, respectively, $\omega$ is the angular velocity of the frame $(x, y, z)$ in Figure 1 referred to inertial space. $M_{B}$ and $\mathrm{m}_{\mathrm{E}}$ are the masses of the satellite body and the beam, respectively, $\mathrm{a}_{0}$ is the acceleration of the frame $(x, y, z)$ referred to inertial space. $T_{B}$ and $T_{E}$ are the external moments acting on the satellite body and the beam, respectively. $F_{B}$ and $F_{E}$ are the external forces acting on the satellite body and the beam, respectively.

\section{Complete Mathematical State Space Model}

So far the beam has been assumed to be rigid, but this is not quite accurate and the vibrations in the flexible beam modify both the moment and force equation. The deflection of the vibrations of the flexible beam modifies the translation equation in (3) with the term (De Lafontaine 1990)

$$
F(x, t)=\sum_{n=1}^{4} p_{n}(x) \ddot{q}_{n}(t)
$$

where $p_{n}$ is called the momentum coefficient and indicates to what extent mode $n$ participates in the translation of the body. The force due to the vibrations of the beam may be substituted into the translation equation of the rigid body to yield the complete translation equation of the spacecraft, viz.

$$
\left(m_{B}+m_{E}\right) a_{0}-m_{B} r_{0} \times \dot{\omega}-m_{E} r_{E} \times \dot{\omega}+\sum_{n=1}^{4} p_{n} \ddot{q}_{n}=F_{B}+F_{E}
$$

The deflection due to the vibrations also influences the moment equation of the spacecraft with the term

$$
T_{y}(x, t)=\sum_{n=1}^{4} h_{n}(x) \ddot{q}_{n}(t)
$$

where $h_{n}$ is called the angular moment coefficient and indicates to what extent mode $\mathrm{n}$ participates in the rotation of the body. Substituting (6) into the rigid body moment equation gives 


$$
J \dot{\omega}+m_{B} r_{0} \times a_{0}+m_{E} r_{E} \times a_{0}+\sum_{n=1}^{4} h_{n} \ddot{q}_{n}=T_{B}+T_{E}
$$

\section{General Equations of Motion}

(6) and (7) may be put into a more general form of equation of motion:

$$
M \ddot{x}+D \dot{x}+K \mathrm{x}=U
$$

where $M$ is the mass matrix and may be written in the form of (9), D is the damping matrix and may be written in the form of (10), $\mathrm{K}$ is the stiffness matrix and may be written in the form of (11). $\mathrm{U}$ is the external forces and moments. The state vector $\mathrm{x}$ is defined as:

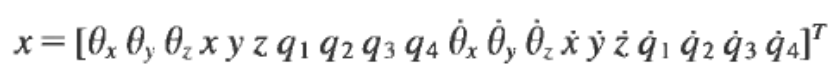

$J_{x B}, J_{y B}, J_{z B}$ represent the moments of inertia of the rigid body. $J_{x E}, J_{y E}, J_{z E}$ represent the moments of inertia of the beam. $M_{B}$ and $m_{E}$ are the masses of the rigid body and the beam, respectively. $\mathrm{H}$ and $\mathrm{p}$ are the angular moment and momentum coefficients, respectively. $Z_{1}-Z_{4}$ are the damping coefficients and $\Omega_{1}-\Omega_{4}$ are the mode frequencies.

$M=\left[\begin{array}{cccccccccc}J_{x B}+J_{x E} & 0 & 0 & 0 & 0 & 0 & 0 & 0 & 0 & 0 \\ 0 & J_{y B}+J_{y E} & 0 & 0 & 0 & m_{B} \frac{a}{2} \div m_{E} \frac{l}{2} & 0 & 0 & 0 & 0 \\ 0 & 0 & J_{z B}+J_{z E} & 0 & -m_{B} \frac{a}{2}+m_{E} \frac{l}{2} & 0 & h_{1 x} & h_{2 x} & h_{3 x} & h_{4 x} \\ 0 & 0 & 0 & m_{B}+m_{E} & 0 & 0 & 0 & 0 & 0 & 0 \\ 0 & 0 & -m_{B} \frac{a}{2}+m_{E} \frac{l}{2} & 0 & m_{B}+m_{E} & 0 & p_{1 y} & p_{2 y} & p_{3 y} & p_{4 y} \\ 0 & m_{B} \frac{a}{2} \div m_{E} \frac{l}{2} & 0 & 0 & 0 & m_{B}+m_{E} & 0 & 0 & 0 & 0 \\ 0 & 0 & h_{1 x} & 0 & p_{1 y} & 0 & m_{E} & 0 & 0 & 0 \\ 0 & 0 & h_{2 x} & 0 & p_{2 y} & 0 & 0 & m_{E} & 0 & 0 \\ 0 & 0 & h_{3 x} & 0 & p_{3 y} & 0 & 0 & 0 & m_{E} & 0 \\ 0 & 0 & h_{4 x} & 0 & p_{4 y} & 0 & 0 & 0 & 0 & m_{E}\end{array}\right]$

$$
D=\left[\begin{array}{cccccccccc}
0 & 0 & 0 & 0 & 0 & 0 & 0 & 0 & 0 & 0 \\
0 & 0 & 0 & 0 & 0 & 0 & 0 & 0 & 0 & 0 \\
0 & 0 & 0 & 0 & 0 & 0 & 0 & 0 & 0 & 0 \\
0 & 0 & 0 & 0 & 0 & 0 & 0 & 0 & 0 & 0 \\
0 & 0 & 0 & 0 & 0 & 0 & 0 & 0 & 0 & 0 \\
0 & 0 & 0 & 0 & 0 & 0 & 0 & 0 & 0 & 0 \\
0 & 0 & 0 & 0 & 0 & 0 & 2 Z_{1} \Omega_{1} m_{E} & 0 & 0 & 0 \\
0 & 0 & 0 & 0 & 0 & 0 & 0 & 2 Z_{2} \Omega_{2} m_{E} & 0 & 0 \\
0 & 0 & 0 & 0 & 0 & 0 & 0 & 0 & 2 Z_{3} \Omega_{3} m_{E} & 0 \\
0 & 0 & 0 & 0 & 0 & 0 & 0 & 0 & 0 & 2 Z_{4} \Omega_{4} m_{E}
\end{array}\right]
$$




$$
\mathbf{K}=\left[\begin{array}{cccccccccc}
0 & 0 & 0 & 0 & 0 & 0 & 0 & 0 & 0 & 0 \\
0 & 0 & 0 & 0 & 0 & 0 & 0 & 0 & 0 & 0 \\
0 & 0 & 0 & 0 & 0 & 0 & 0 & 0 & 0 & 0 \\
0 & 0 & 0 & 0 & 0 & 0 & 0 & 0 & 0 & 0 \\
0 & 0 & 0 & 0 & 0 & 0 & 0 & 0 & 0 & 0 \\
0 & 0 & 0 & 0 & 0 & 0 & 0 & 0 & 0 & 0 \\
0 & 0 & 0 & 0 & 0 & 0 & \Omega_{1}^{2} m_{E} & 0 & 0 & 0 \\
0 & 0 & 0 & 0 & 0 & 0 & 0 & \Omega_{2}^{2} m_{E} & 0 & 0 \\
0 & 0 & 0 & 0 & 0 & 0 & 0 & 0 & \Omega_{3}^{2} m_{E} & 0 \\
0 & 0 & 0 & 0 & 0 & 0 & 0 & 0 & 0 & \Omega_{4}^{2} m_{E}
\end{array}\right]
$$

It is also possible to bring the system into a standard state space form by premultiplying with the inverse of the mass matrix on both sides, viz.

$$
\begin{aligned}
& \dot{x}=A x+B u \\
& y=C x+D u
\end{aligned}
$$

and

$$
A=\left[\begin{array}{cc}
0 & I \\
-M^{-1} K & -M^{-1} D
\end{array}\right] \quad B=\left[\frac{0}{M^{-1}}\right]
$$

(12) represents the complete mathematical model of the spacecraft assuming four vibration frequencies and is simulated using different types of excitations, as described in section 5. An accelerometer is connected to the end of the beam and represents the measurements used in the frequency identification.

\section{Identification of Vibration Frequencies using Nonparametric Methods}

Nonparametric methods are based on Fourier analysis and identify the frequency contents in a signal, i.e. estimate the power spectral density (PSD) of a signal. Spectral estimation may be divided into two main groups:

* direct methods

* indirect methods

Each main group can be further subdivided into different methods. In this paper we will only look at the following direct methods: the Periodogram and the Welch method. Direct methods are based on the definition

$$
P_{y y}(f)=\lim E\left[\frac{1}{\underset{M}{2 M+\infty} \mid} \mid \sum_{k=-M}^{M} y(k) e^{-\left.j 2 \Pi f k\right|^{2}}\right]
$$

where $y(k)$ is the measurement and $P_{y y}(f)$ is the PSD. by

Using the Wiener-Khintchine theorem, an alternative formula for the PSD is given

$$
P_{y y}(f)=\sum_{k=-\infty}^{\infty} r_{y y}(k) e^{-j 2 \Pi f k} \quad-1 / 2 \prec f \prec 1 / 2
$$

where $r_{y y}(k)$ is the autocorrelation.

Periodogram

The periodogram spectral estimator relies on the definition of the spectral density 
given by (13). By neglecting the expectation operator $\mathrm{E}$ and using only the available data $[y(0), y(1) \ldots y(N-1)]$, the periodogram spectral estimator may be defined as (Kay 1988)

$$
\hat{P}_{p e r}(f)=\frac{1}{N}\left|\sum_{k=0}^{N-1} y(k) e^{-j 2 \Pi f k}\right|^{2}
$$

An alternative formula for the estimated periodogram based on (14) is

$$
\hat{P}_{p e r}(f)=\sum_{k=-(N-1)}^{N-1} \hat{r}_{y y}(k) e^{-j 2 \Pi f k}
$$

where $\hat{r}_{y y}(k)$ is the estimated autocorrelation.

Before deciding to use a PSD estimator, it is natural to ask: "What properties does this method have? Does it give accurate estimates, etc?" One important property is that the estimator should be consistent. In the general PSD case this means that

$$
\hat{P}(\mathrm{f}) \Rightarrow \mathrm{P}_{\mathrm{yy}}(\mathrm{f}) \text { as } \mathrm{N} \Rightarrow \infty
$$

i.e. the estimated PSD becomes the true PSD as the number of measurements $\mathrm{N}$ tends to infinity. Consistency requires that the bias $\rightarrow 0$ as $\mathrm{N} \rightarrow \infty$ and thus the expectation or mean of the estimated PSD becomes the true PSD. In addition a "reliable" estimator requires a low mean-square error (MSE). If the bias $\rightarrow 0$, the MSE of the estimator $\rightarrow$ the variance. Hence, a PSD estimator is described by its expectation and variance.

The expectation of the periodogram is given in (17).

$$
\begin{aligned}
E\left[\hat{P}_{p e r}(f)\right] & =\sum_{k=-(N-1)}^{N-1}\left[1-\frac{|k|}{N}\right] r_{y y}(k) e^{-j 2 \pi f \mathrm{k}} \\
& =\sum_{k=-(N-1)}^{N-1} w_{B}(k) r_{y y}(k) e^{-j 2 \pi f k} \\
& =W_{B}(f) * P_{y y}(f)
\end{aligned}
$$

${ }^{1} \mathrm{~W}_{\mathrm{B}}(\mathrm{f})$ is called a spectral window and is the Fourier transformation of the lag window $\mathrm{w}_{\mathrm{B}}(\mathrm{k})$. From (17) it may be seen that if $\mathrm{w}_{\mathrm{B}}(\mathrm{k}) \rightarrow 1$, then $E\left[\hat{P}_{p e r}(f)\right] \rightarrow P_{y y}(\mathrm{f})$. Furthermore, $\mathrm{w}_{\mathrm{B}}(\mathrm{k}) \rightarrow 1$ as $\mathrm{N} \rightarrow \infty$.

Thus the periodogram is biased in general for finite data records, but its expectations tend to the true value as $\mathrm{N} \rightarrow \infty$. It can be shown that the variance is given by (Kay 1988)

$$
\operatorname{Var}\left[\hat{P}_{p e r}(f)\right]=P_{y y}^{2}(f)\left[1+\left[\frac{\sin 2 \Pi f N}{N \sin 2 \Pi f}\right]^{2}\right]
$$

(18) shows that the variance is of the order $P_{y y}^{2}$, i.e. it is constant and independent of $\mathrm{N}$. Since the periodogram is in general biased and the variance does not decrease as $\mathrm{N} \rightarrow \infty$, the periodogram is not a consistent estimator. However, the periodogram has some desirable properties and some unwanted properties, summarised below.

* Noisy measurements often show large fluctuations.

* Due to large sidelobes, weak signal components may be masked by strong signal components.

* in (17) denotes the convolution. 
* Good resolutions for signal components with nearly equal amplitude.

* The resolution is $\approx 1 / \mathrm{N}$, and no other nonparametric method can equal this.

\section{Welch Spectral Estimator}

Welch divides the available data into $\mathrm{K}$ independent data sets (segments) of length $\mathrm{L}$, and uses a lag window for each data set. The data sets overlap. The lag window reduces the sidelobe level. By overlapping the data sets, usually by $50 \%-70 \%$, some extra variance reduction is achieved. Welch's spectral estimator may be written (Kay 1988)

$$
\hat{P}_{W}(f)=\frac{1}{K} \sum_{m=0}^{K-1} \hat{P}_{M}^{(m)}(f)
$$

where

$$
\hat{P}_{M}^{(m)}(f)=\frac{1}{U L} \mid \sum_{k=0}^{L-1} w(k) y_{m}(k) e^{-\left.j 2 \Pi f k\right|^{2}}
$$

$\mathrm{K}$ is the number of segments, $\mathrm{L}$ is the length of each segment, w(k) is the lag window of length $L, U$ is averaging of the lag window, $y_{m}(k)$ is the measurements and $N$ is the total number of data. The expectation may be shown to be (Bøe, 1991)

$$
E\left[\hat{P}_{W}(f)=\frac{1}{U N} P_{y v}(f) *|W(f)|^{2}\right.
$$

The variance is roughly inversely proportional to the number of segments

$$
\operatorname{Var}\left[\hat{P}_{w}(f)\right]=\frac{1}{K} P_{y y}^{2}(f)
$$

Welch's spectral estimator is a consistent estimator with the following properties:

* Probably the most commonly used nonparametric method today.

* Welch's spectral estimator is a consistent estimator.

* Overlapping data sets reduce the variance by less than $1 / \mathrm{K}$, if no overlapping is used the variance is reduced by $1 / \mathrm{K}$.

* Reduced resolution due to the windowing.

\section{Identification of Vibration Frequencies using Parametric Methods}

Parametric methods identify unknown parameters in a chosen mathematical model. Among the best known models are the AR (autoregressive), ARX (autoregressive with external input), ARMA (autoregressive moving average) and ARMAX (autoregressive moving average with external input). The procedure with parametric methods is as follows:

a. Choose a mathematical model for the process to be identified.

b. Choose an identification method that suits the chosen method.

Different identification methods are normally available for each model; the simplest and fastest methods are the analytical methods, i.e. parameter estimation without using a numerical search procedure. Analytical methods may be suboptimal, however, depending on the SNR (signal-to-noise ratio), they often give accurate parameter estimates and they are fast. Numerical search methods may also be used to estimate the unknown parameters. Ljung (1987) claims that on the average a Gauss-Newton 
numerical search method performs best among the numerical methods. Numerical search methods are slow but they may give more accurate parameter estimates than analytical methods, especially for medium high and low SNR measurements. Definition of medium high and low SNR measurements will be given in section 5 .

\section{AR (AutoRegressive) Methods}

AR methods are a class of methods used to determine the unknown parameters in an AR model. Before describing AR methods, the AR model will be given.

An AR model is simply a difference equation of the following form

$$
y(k)+a_{1} y(k-1)+a_{2} y(k-2)+\ldots a_{p} y(k-p)=e(k)
$$

or in the more compact form

$$
A\left(q^{-1}\right) y(k)=e(k)
$$

where

$$
A\left(q^{-1}\right)=1+a_{1} q^{-1}+a_{2} q^{-1}+\ldots a_{p} q^{-p}
$$

and $y(k)$ is the output at time $k$, whereas $e(k)$ is the disturbance or residual and is assumed to be a zero-mean stochastic process with a rational spectral density.

The parameters in the AR model may be identified using different methods such as linear regression, least squares estimates or prediction error methods.

\section{Linear regression}

The linear regression method for the AR model may be expressed as:

$$
y(k)=\phi^{T}(k) \hat{\theta}+e(k)
$$

where

$$
\begin{aligned}
\phi(k) & =(-y(k-1),-y(k-2), \ldots-y(k-p))^{T} \\
\hat{\theta} & =\left(a_{1}, a_{2}, \ldots a_{p}\right)^{T}
\end{aligned}
$$

and where $\mathrm{p}$ is the number of unknown parameters. Ignoring the noise term $\mathrm{e}(\mathrm{k})$, which normally is unknown, and performing a small modification of (24) we obtain

$$
y(k)=\Phi \hat{\theta}
$$

and

$$
\Phi=\left(\phi(k)^{T}, \phi(k+1)^{T}, \ldots \phi(N)^{T}\right)^{T}
$$

(25) may be written in component form as

$$
\left[\begin{array}{c}
y(k) \\
y(k+1) \\
\vdots \\
y(N)
\end{array}\right]=\left[\begin{array}{cccc}
-y(k-1) & -y(k-2) & \ddots & -y(k-p) \\
-y(k) & -y(k-1) & \cdots & -y(k+1-p) \\
\cdot & \ddots & \cdots & \vdots \\
-y(N-1) & -y(N-2) & \cdots & -y(N-p)
\end{array}\right]\left[\begin{array}{c}
\hat{a}_{1} \\
\hat{a}_{2} \\
\cdot \\
\hat{a}_{p}
\end{array}\right]
$$

(26) forms a set of linear equations and the parameter vector $\hat{\theta}$ can be determined by taking the inverse of $\Phi$. see (25) or (26). Often (26) consists of more equations than unknown parameters and can be solved as a set of overdetermined equations. 


\section{Least Squares Estimate}

Least squares estimate normally introduces the equation error and form the quadratic loss function shown in (27).

$$
L(\theta)=\frac{1}{N} \sum_{k=1}^{N} \frac{1}{2} \varepsilon^{2}(k)
$$

where the equation error is: $\varepsilon(k)=y(k)-\phi^{T} \theta$.

The parameter vector which minimizes (27) is given by

$$
\hat{\theta}=\left(\Phi^{T} \Phi\right)^{-1} \Phi^{T} Y
$$

where

$$
Y=[y(1) y(2) \ldots y(N)]^{T}
$$

If $\Phi$ is a $N \times p$ matrix of rank p then the expression $\left(\Phi^{T} \Phi\right)^{-1} \Phi^{T}$ in (28) is the pseudoinverse of $\Phi$.

(28) requires an inversion and a model form that may be easier to solve is (29).

$$
\hat{\theta}=\left[\frac{1}{N} \sum_{k=1}^{N} \phi(k) \phi^{T}(k)\right]^{-1}\left[\frac{1}{N} \sum_{k=1}^{N} \phi(k) y(k)\right]
$$

(29) may be viewed as a correlation function and may be easier to implement than (28).

Many text books give (29) in another form

$$
R_{y y} \hat{\theta}=r_{y y}
$$

known as the normal equation, where

$$
\begin{aligned}
R_{y y}(N) & =\frac{1}{N} \sum_{k=1}^{N} \phi(k) \phi^{T}(k) \\
r_{y y}(N) & =\frac{1}{N} \sum_{k=1}^{N} \phi(k) y(k)
\end{aligned}
$$

\section{Prediction Error Methods}

Prediction error methods can be considered to be a generalization of the least squares methods. The parameter estimates are determined by minimizing a suitable loss function. Many estimation methods make use of the prediction error to estimate the unknown parameters, and the quadratic loss function (27), shown for the LS case, is often used with different modifications. However, a more complex loss function may also be used (Söderström et al. 1989).

\section{Autocorrelation Method}

The autocorrelation method can be intepreted as a prediction error method where the AR parameters are estimated by minimizing the loss function (Kay 1988)

$$
L(\theta)=\frac{1}{N} \sum_{k=-\infty}^{\infty}|\varepsilon(k)|^{2}
$$

where $\mathrm{N}$ is the number of measurements. The prediction errors are calculated from

$$
\varepsilon(k)=y(k)-\hat{y}(k)
$$




$$
\hat{y}(k)=\sum_{l=1}^{p} \hat{a}(l) y(k-l)
$$

The loss function (31) is minimized by differentiating with respect to the parameter vector and the solution may be expressed in matrix form (Kay 1988)

$$
\left[\begin{array}{cccc}
\hat{r}_{y y}(0) & \hat{r}_{Y Y}(-1) & \ldots & \hat{r}_{y y}(-(p-1)) \\
\hat{r}_{y y}(1) & \hat{\mathbf{r}}_{Y Y}(0) & \ldots & \hat{r}_{y y}(-(p-2)) \\
\cdot & \cdot & \cdots & \cdot \\
\cdot & \cdot & \cdot & \cdot \\
\hat{r}_{y y}(p-1) & \hat{r}_{Y Y}(p-2) & \cdots & \hat{r}_{y y}(0)
\end{array}\right]\left[\begin{array}{c}
\hat{a}_{1} \\
\hat{a}_{2} \\
\cdot \\
\cdot \\
\hat{a}_{p}
\end{array}\right]=-\left[\begin{array}{c}
\hat{r}_{y y}(1) \\
\hat{r}_{y y}(2) \\
\cdot \\
\cdot \\
\hat{r}_{y y}(p)
\end{array}\right]
$$

where the covariance elements are estimated from

$$
\hat{r}_{y y}(k)=\left\{\begin{array}{l}
\frac{1}{N} \sum_{l=0}^{N-1-k} y^{*}(l) y(l+k) \quad \text { for } \quad k=0,1 \ldots p \\
\hat{r}_{y y}^{*}(-k) \text { for } k=-(p-1),-(p-2) \ldots-1
\end{array}\right.
$$

Only $\mathrm{y}(1) \neq 0$ is used in calculating $\hat{r}_{y y}(k)$.

It is also possible to estimate the AR parameters directly from the data matrix as described in (26) and in that case (26) takes the form

$$
\left[\begin{array}{cccc}
0 & \dot{0} & 0 \\
-y(0) & 0 & \cdots & 0 \\
-y(1) & -y(0) & \cdots & 0 \\
\cdot & \cdot & \cdots & \cdot \\
-y(p) & -y(p-1) & \cdots & -y(0) \\
-y(N-1) & -y(N-2) & \cdots & -y(N-p-1) \\
0 & -y(N-1) & \cdots & -y(N-p) \\
\dot{0} & 0 & . & -y(N-1)
\end{array}\right]\left[\begin{array}{c}
\hat{a}_{1} \\
\hat{a}_{2} \\
\cdot \\
\cdot \\
\hat{a}_{p}
\end{array}\right]=\left[\begin{array}{c}
y(0) \\
y(1) \\
y(2) \\
\cdot \\
\cdot \\
y(N+p-1)
\end{array}\right]
$$

or, more compactly

$$
Y a ̂=y
$$

Therrien (1992) makes use of the data matrix $\mathrm{Y}$ and formulates an equation known as the Yule-Walker equation, viz.

$$
\left(Y^{H} Y\right) \hat{a}=\left[\begin{array}{l}
\hat{S} \\
0
\end{array}\right]
$$

$\hat{S}$ is the estimate of the sum of the squared errors and is normally calculated simultaneously with the $\hat{a}$ parameters. The $\mathrm{Y}$ - and $\hat{a}$-matrices in (34) and (35) are equal except for the first row in the Y-matrix of (35) which is removed, whereas $\hat{a}=\left[1, \hat{a}_{1}, \hat{a}_{2}, \ldots \hat{a}_{p}\right]^{T}$.

Summary of the autocorrelation method

* This method is also called a windowed method because it replaces the unknown initial values by zeros.

* The windowing reduces the resolution.

* This method is not recommended for short data records.

* Poles close to the unit circle decrease accuracy of the parameter estimates. 


\section{Covariance Method}

The covariance estimator may be found by minimizing the loss function (Kay 1988)

$$
L(\theta)=\frac{1}{N-p} \sum_{k=p}^{N-1}|\varepsilon(k)|^{2}
$$

where $\varepsilon(k)$ is defined in (31).

Note that the only difference between the covariance method and the autocorrelation method is the range of summation. In the covariance method all data points needed to estimate the parameter vector have been observed, and no zeroing of data is required. The solution to (36) may be written

$$
\left[\begin{array}{cccc}
\hat{r}_{y y}(1,1) & \hat{r}_{y y}(1,2) & \ldots & \left.\hat{r}_{y y}(1, p)\right) \\
\hat{r}_{y y}(2,1) & \hat{r}_{y y}(2,2) & \ldots & \left.\hat{r}_{y y}(2, p)\right) \\
\cdot & \cdot & . & \cdot \\
\cdot & \cdot & . & \cdot \\
\hat{r}_{y y}(p, 1) & \hat{r}_{y y}(p, 2) & . & \hat{r}_{y y}(p, p)
\end{array}\right]\left[\begin{array}{c}
\hat{a}_{1} \\
\hat{a}_{2} \\
\cdot \\
\cdot \\
\hat{a}_{p}
\end{array}\right]=-\left[\begin{array}{c}
\hat{r}_{y y}(1,0) \\
\hat{r}_{y y}(2,0) \\
\cdot \\
\cdot \\
\hat{r}_{y y}(p, 0)
\end{array}\right]
$$

where

$$
\hat{r}_{y y}(j, k)=\frac{1}{N-p} \sum_{n=p}^{N-1} y^{*}(n-j) y(n-k)
$$

It is also possible to estimate the AR parameters directly from a data matrix given by (38).

$$
\left[\begin{array}{cccc}
-y(p-1) & -y(p-2) & \ldots & -y(0) \\
-y(p) & -y(p-1) & \ldots & -y(1) \\
\cdot & \cdot & . & \cdot \\
-y(N-2) & -y(N-3) & . & -y(N-1-p)
\end{array}\right]\left[\begin{array}{c}
\hat{a}_{1} \\
\hat{a}_{2} \\
\cdot \\
\cdot \\
\hat{a}_{p}
\end{array}\right]=\left[\begin{array}{c}
y(p) \\
y(p+1) \\
\cdot \\
\cdot \\
y(N-1)
\end{array}\right]
$$

From (38) it is easy to see that the covariance method makes use of measured data only. It is also possible to form a Yule-Walker equation like that in (35).

Summary of the covariance method

* The estimated poles, using the covariance method, are not guaranteed to lie within the unit circle.

* For data consisting of pure sinusoids, the covariance method may be used to extract the frequencies perféctly.

\section{Modified Covariance Method or Forward-Backward Method}

This method provides twice as much data upon which the identification can be based and thereby reduces the variance. The modified covariance method identifies the AR parameters by minimizing the average of the forward and backward loss function (Kay 1988)

$$
L(\theta)=\frac{1}{2}\left(L^{f}(\theta)+L^{b}(\theta)\right)
$$


where

$$
\begin{aligned}
L^{f}(\theta) & =\frac{1}{N-p} \sum_{k=p}^{N-1}\left|y(k)+\sum_{l=1}^{p} \hat{a}(l) y(k-l)\right|^{2} \\
L^{b}(\theta) & =\frac{1}{N-p} \sum_{k=0}^{N-1-p}\left|y(k)+\sum_{l=1}^{p} \hat{a}^{*}(l) y(k+l)\right|^{2}
\end{aligned}
$$

As for the covariance method, summations are over the prediction errors that involve measured data samples only. The solution for the modified covariance method is given by (37), but the definition of $\hat{r}_{y y}(j, k)$ now takes the form

$$
\hat{r}_{y y}(j, k)=\frac{1}{2(N-p)}\left(\sum_{n=p}^{N-1} y^{*}(n-j) y(n-k)+\sum_{n=0}^{N-1-p} y(n+j) y^{*}(n+k)\right)
$$

The AR parameters may also be calculated directly from a data matrix. This data matrix is composed of both the forward and the backward data matrix and has the form

$$
\left[\begin{array}{cccc}
-y(p-1) & -y(p-2) & \ldots & -y(0) \\
-y(p) & -y(p-1) & \cdots & -y(1) \\
\cdot & \cdot & \cdots & \cdot \\
-y(N-2) & -y(N-3) & \cdots & -y(N-1-p) \\
-y(N-p)^{*} & -y(N-p+1)^{*} & \cdots & -y(N-1)^{*} \\
-y(N-p-1)^{*} & -y(N-p)^{*} & \cdots & -y\left(N-2^{*}\right) \\
\cdot & \cdot & \cdots & \cdot \\
-y(1)^{*} & -y(2)^{*} & \cdots & -y(p)^{*}
\end{array}\right]\left[\begin{array}{c}
\hat{a}_{1} \\
\hat{a}_{2} \\
\cdot \\
\cdot \\
\hat{a}_{p}
\end{array}\right]=\left[\begin{array}{c}
y(p) \\
y(p+1) \\
\cdot \\
\cdot \\
y(N-1) \\
y(N-p-1)^{*} \\
\cdot \\
y(0)^{*}
\end{array}\right]
$$

where $y()^{*}$ for complex data is the complex conjugate and $y()^{*}$ for real data $=y()$. It is also possible to form Yule-Walker-like equations from the forward-backward data matrix as shown in (41), (Therrien 1992)

$$
\left(Y^{H} Y+\tilde{Y}^{T} \tilde{Y}^{*}\right) \hat{a}=\left[\begin{array}{c}
\hat{S}_{f b} \\
0
\end{array}\right]
$$

The backward correlation matrix $\tilde{Y}^{T} \tilde{Y}^{*}$ is the reversal of the forward correlation matrix $Y^{H} Y$.

Summation of the modified covariance method

* The modified covariance method is also called the forward-backward method.

* Due to the calculation of both a forward and backward estimator, which involve more data, the modified covariance method gives accurate estimates.

* The modified covariance method does not guarantee the estimated poles to lie inside the unit circle, but they usually do.

* The modified covariance method is suitable for sinusoidal components in white noise.

* The method can be recommended for short data records.

\section{ARX (AutoRegressive with eXogeneous input) Methods}

AR methods identify parameters in a time series model, i.e. a difference equation 
without deterministic inputs. It is possible to modify that model to include deterministic inputs, which leads to an ARX model. Methods to identify parameters in an ARX model are called ARX methods. Since an ARX model is an AR model with deterministic input, (22) modifies to

$$
\begin{array}{r}
y(k)+a_{1} y(k-1)+\ldots a_{p} y(k-p)=b_{1} u(k-1)+b_{2} u(k-2) \\
+\ldots b_{m} u(k-m)+e(k)
\end{array}
$$

or, more compactly

$$
A\left(q^{-1}\right) y(k)=B\left(q^{-1}\right) u(k)+e(k)
$$

where

$$
\begin{aligned}
& A\left(q^{-1}\right)=1+a_{1} q^{-1}+a_{2} q^{-2}+\ldots a_{p} q^{-p} \\
& B\left(q^{-1}\right)=b_{1} q^{-1}+b_{2} q^{-2}+\ldots b_{m} q^{-m}
\end{aligned}
$$

The unknown parameters in the $\mathrm{A}\left(\mathrm{q}^{-1}\right)$ and $\mathrm{B}\left(\mathrm{q}^{-1}\right)$ polynomials may be found by utilizing many of the same identification methods as described for the AR model, but with some modifications. The $\mathrm{A}\left(\mathrm{q}^{-1}\right)$ and $\mathrm{B}\left(\mathrm{q}^{-1}\right)$ polynomials can be determined from the linear regression method described for $A R$ models, but with the following modifications:

$$
\begin{aligned}
\phi(k) & =(-y(k-1) \ldots y(k-p) u(k-1) \ldots u(k-m))^{T} \\
\hat{\theta} & =\left(a_{1}, a_{2}, \ldots a_{p}, b_{1}, b_{2}, \ldots b_{m}\right)^{T}
\end{aligned}
$$

Componentwise (26) will modify to

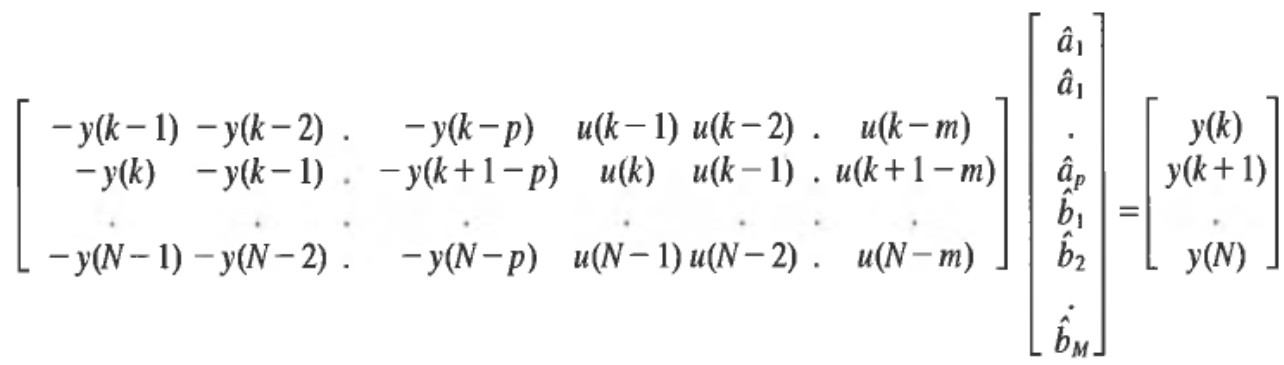

It is also possible to apply either a modified autocorrelation or covariance method or other methods which can be solved both for the $\hat{a}$ and the $\hat{b}$ parameters.

\section{Instrumental Variable (IV) Methods}

The least square method is consistent as long as the disturbance is white. It is, however, possible to make a consistent LS estimator even if the disturbance is not white. This leads to the IV method which may be written as

$$
\hat{\theta}=\left[\frac{1}{N} \sum_{k=1}^{N} z(k) \phi^{T}(k)\right]^{-1}\left[\frac{1}{N} \sum_{k=1}^{N} z(k) y(k)\right]
$$

The $\mathrm{z}(\mathrm{k})$ matrix is called the instrument and should be uncorrelated with the disturbance. 
IV methods are usually applied in connection with ARX models with non-white disturbances.

ARMA (AutoRegressive Moving Average) and ARMAX (AutoRegressive Moving Average with eXogeneous input) Methods

These methods identify parameters in ARMA and ARMAX models. These models handle processes which cannot be modelled as pure AR or ARX. Since nearly all data are corrupted by some amount of measurement noise, the nonlinear search methods used for ARMA and ARMAX are often the appropriate ones. Unfortunately, no fast and effective method for estimating parameters from these nonlinear methods exist, and as an alternative, many suboptimal but easily implementable algorithms have been proposed. Most suboptimal methods make separate estimates of the AR and MA parameters. Typically, the AR parameters are estimated first and then used to estimate the MA parameters. The difference equation of an ARMAX model may be written as

$$
\begin{aligned}
y(k)+a_{1} y(k-1)+\ldots a_{p} y(k-p)= & b_{1} u(k-1)+b_{2} y(k-2) \\
& +\ldots b_{m} u(k-m)+e(k) \\
& +c_{1} e(k-1)+\ldots c_{q} y(k-q)
\end{aligned}
$$

(47) can be written more compactly as

$$
A\left(q^{-1}\right) y(k)=B\left(q^{-1}\right) u(k)+C\left(q^{-1}\right) e(k)
$$

where

$$
\begin{aligned}
& A\left(q^{-1}\right)=1+a_{1} q^{-1}+a_{2} q^{-2}+\ldots a_{p} q^{-p} \\
& B\left(q^{-1}\right)=b_{1} q^{-1}+b_{2} q^{-2}+\ldots b_{m} q^{-m} \\
& C\left(q^{-1}\right)=1+c_{1} q^{-1}+c_{2} q^{-2}+\ldots c_{q} q^{-q}
\end{aligned}
$$

An ARMA model is the special case of (47) or (48) where the deterministic input $u(k)$ is zero.

Instead of estimating ARMA and ARMAX parameters by suboptimal analytical methods, it is common to estimate all parameters directly from a numerical search routine. Numerical search methods may give better results than the previously described methods (Ljung 1987), but, unfortunately, the computation is much higher. Numerical iterative search methods are often used in connection with prediction error formulations. Söderström et al. (1989) show that the results of a certain prediction error method (PEM) become equal to the results of the maximum likelihood (ML) method when the disturbances are Gaussian distributed.

Ljung (1987) shows that the solution of a numerical search routine which minimizes the quadratic loss function given in (27) may be written as

$$
\hat{\theta}_{N}^{(i+1)}=\hat{\theta}_{N}^{(i)}-\mu_{N}^{(i)}\left(R_{N}^{(i)}\right)^{-1} L_{N}^{\prime}\left(\hat{\theta}_{N}^{(i)}\right)
$$

where

$\hat{\theta}_{N}^{(i)} \quad$ denotes the $\mathrm{i}^{\text {th }}$ iteration

$L_{N}^{\prime}\left(\hat{\theta}_{N}^{(i)}\right)$ is the gradient of $L_{N}(\hat{\theta})$

$R_{N}^{(i)} \quad$ modifies the search direction

$\mu_{N}^{(i)} \quad$ is the step size

$R_{N}^{(i)}$ and $L_{N}^{\prime}\left(\hat{\theta}_{N}^{(i)}\right)$ together determine the search direction whereas $\mu_{N}^{(i)}$ denotes the 
numerical step length. The gradient of the performance criterion, (27), may be calculated from

$$
L_{n}^{\prime}(\hat{\theta})=-\frac{1}{N} \sum_{k=1}^{N} \Psi(k) \varepsilon(k)
$$

Söderström et al. (1989) show that the gradient matrix $\Psi$ can be calculated from

$$
\begin{aligned}
\Psi(k)=\frac{d}{d \theta} \varepsilon(k)= & -\left(y_{f}(k-1) \ldots y_{\mathrm{f}}(k-p)-u_{f}(k-1) \ldots-u_{f}(k-m)\right. \\
& \left.-\varepsilon_{f}(k-1) \ldots-\varepsilon_{f}(k-q)\right)
\end{aligned}
$$

where $\mathrm{y}_{\mathrm{f}}(\mathrm{k}), \mathrm{u}_{\mathrm{f}}(\mathrm{k})$ and $\varepsilon_{\mathrm{f}}(\mathrm{k})$ are $\mathrm{y}(\mathrm{k}), \mathrm{u}(\mathrm{k})$ and $\varepsilon(\mathrm{k})$, respectively, filtered by $1 / \mathrm{C}\left(\mathrm{q}^{-1}\right)$, i.e.,

$$
\begin{aligned}
& y_{f}(k)=\frac{1}{C\left(q^{-1}\right)} y(k) \\
& u_{f}(k)=\frac{1}{C\left(q^{-1}\right)} u(k) \\
& \varepsilon_{f}(k)=\frac{1}{C\left(q^{-1}\right)} \varepsilon(k)
\end{aligned}
$$

If the Gauss-Newton method is used, (Ljung (1987) recommends Gauss-Newton), the following form for $\mathrm{R}_{\mathrm{N}}$ may be used:

$$
R_{N}=\frac{1}{N} \sum_{k=1}^{N} \Psi(k) \Psi^{T}(k)
$$

\section{Recursive Identification Methods}

Previous sections have dealt with different batch methods, also called off-line methods. These methods require that all data is recorded in a batch and, hence, cannot be used in real-time applications. Real-time (on-line) methods can be used in real time applications and may also be used to track time-varying parameters. Many recursive identification methods can be derived as approximations to batch methods. The results are, however, often a reduction in accuracy (Landau 1990). Recursive methods handle both AR, ARX, ARMA and ARMAX models.

\section{Recursive Least Squares with Forgetting Factor}

The loss function is modified as (Ljung 1987)

$$
L(\theta)=\sum_{s=1}^{k} \lambda^{k-s} \varepsilon^{2}(k)
$$

where $\lambda$ is the forgetting factor, usually taken to be between 0.95 and 0.99 .

With increasing $\mathrm{k}$ the information in the previous data is forgotten. The smaller $\lambda$ is the quicker the information will be forgotten. Performing the minimization of (53), (Ljung 1987), we obtain

$$
\begin{aligned}
\hat{\theta} & =\hat{\theta}(k-1)+K(k) \varepsilon(k) \\
K(k) & =\frac{P(k-1) \varphi(k)}{\lambda+\varphi^{T}(k) P(k-1) \varphi(k)} \\
\varepsilon(k) & =y(k)-\varphi^{T}(k) \hat{\theta}(k-1)
\end{aligned}
$$




$$
P(k)=\frac{P(k-1)-\frac{P(k-1) \varphi(k) \varphi^{T}(k) P(k-1)}{\lambda+\varphi^{T}(k) P(k-1) \varphi(k)}}{\lambda}
$$

The forgetting factor makes the RLS algorithm capable of tracking time-varying parameters. Another popular recursive identification method is the pseudolinear regression.

\section{Recursive Pseudolinear Regression}

This method is also called the extended least squares or the approximate ML method and the formulae are given by Söderström et al. (1989), viz.

$$
\begin{aligned}
\hat{\theta} & =\hat{\theta}(k-1)+K(k) \varepsilon(k) \\
\varepsilon(k) & =y(k)-\varphi^{T}(k) \hat{\theta}(k-1) \\
P(k) & =P(k-1)-\frac{P(k-1) \varphi^{T}(k) P(k-1)}{1+\varphi^{T}(k) P(k-1) \varphi(k)} \\
K(k) & =\frac{P(k-1) \varphi(k)}{1+\varphi^{T}(k) P(k-1) \varphi(k)}
\end{aligned}
$$

\section{Simulation Results}

The spacecraft model described in section 2 is simulated. The spacecraft is composed of a flexible beam connected to a rigid satellite core. The flexible beam is modelled as a 4-mode Euler-Bernoulli beam and the vibration frequencies of the flexible beam are given in Table 1 . The goal is to identify these frequencies directly or to identify parameters so that the frequencies can be determined. The process is limited to contain measurement noise only and the measurement noise is assumed to be white, i.e. Gaussian distributed white noise. Nonparametric methods estimate the power spectrum and, thus, estimate the vibration frequencies directly. Parametric methods identify the structure parameters from which the frequencies may be calculated. The sample interval was fixed to $0.04 \mathrm{sec}$. during the simulations. All simulations are performed using MATLAB.

\section{Excitation of the Structure Vibrations}

Disturbances or attitude corrections may force the flexible parts of the spacecraft to vibrate. Although different types of excitations may be considered, for the simulations reported here, the excitation was to be limited to a unit step torque. The torque works around the $\mathrm{z}$-axis (cf. Figure 1). An impulse torque and a unit step torque give very similar vibration patterns (Skullestad 1995). It is important that the excitation signal excites all vibration modes to be identified, as those vibration modes which are not sufficiently excited (persistently excited) cannot be identified.

\section{Measurements}

The vibration modes/parameters may be identified using measurements from different sensors. An accelerometer, a strain-gauge, or a remote attitude measurement sensor are all very possible sensors. In this paper an accelerometer will be used as the measurement sensor. Different levels of white measurement noise are added to the accelerometer signal. The level of the measurement noise will be denoted by the 

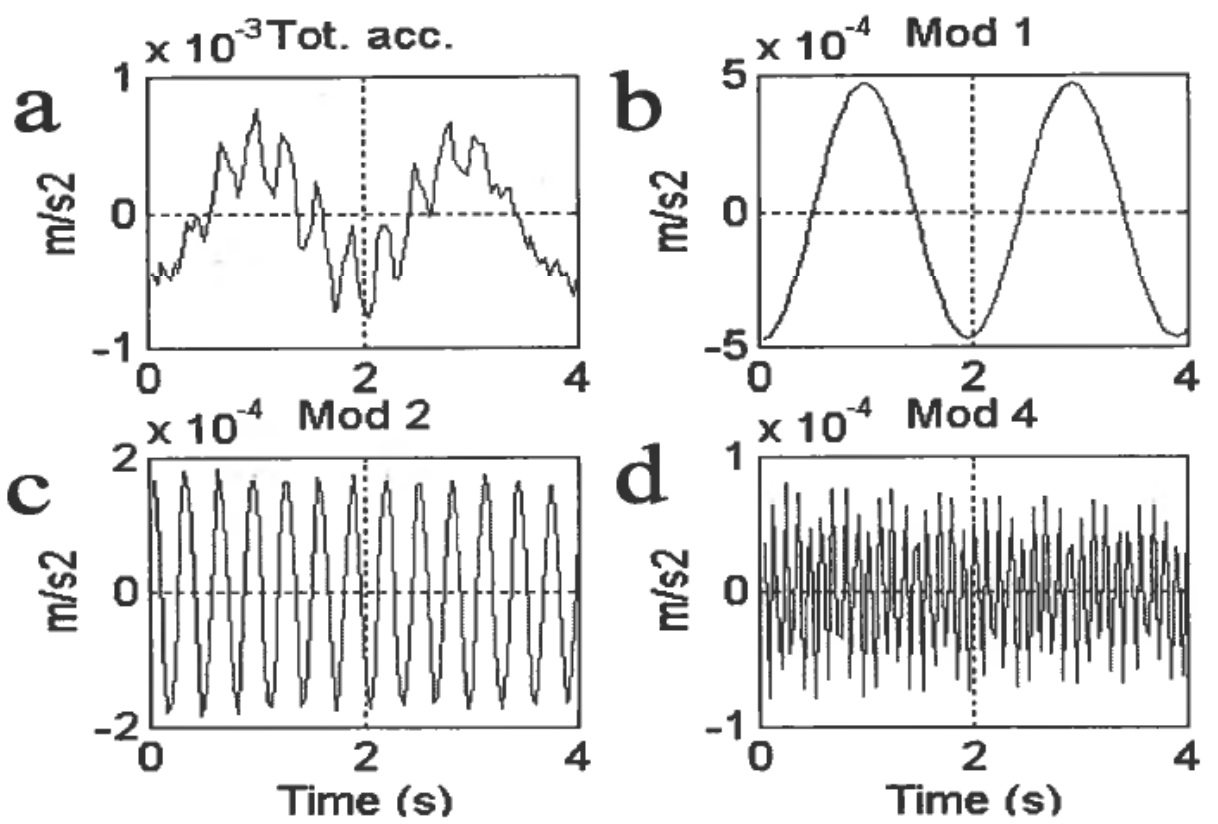

Figure 4a,b,c,d. Measured acceleration without noise.

standard deviation. The spacecraft is excited by a unit torque around the z-axis. If all measurements are considered to be noise-free, Figure 4 shows the total acceleration at the end of the beam due to all vibration modes, and in addition the acceleration due to the individual vibration modes 1,2 and 4 . The acceleration due to vibration mode 3 is similar to vibration mode 2 and is not shown.

The noise and error-free accelerometer output is numerically equal to the second derivative (with respect to time) of the displacement of the beam at the accelerometer location multiplied with a scaling factor $\mathrm{K}_{\mathrm{ACC}}$, viz,

$$
a_{a c c}(t)=K_{a c c} \frac{\partial^{2} \mu(x, t)}{\partial t^{2}}=K_{a c c} \sum_{n=1}^{4} \frac{\partial^{2} q_{n}(t)}{\partial t^{2}} W_{n}(x)
$$

Gaussian white noise is added to the measurement shown in figure $4 \mathrm{a}$ and the resulting noise corrupted measurement signal is then considered to be the basic input for the identification methods that have previously been described.

\subsection{Frequency Identification using Nonparametric Methods}

Nonparametric methods estimate the power spectral density (PSD) directly. The three most important external parameters which mainly determine the accuracy of the identified frequencies/parameters are the level of the measurement noise (the signal-to-noise ratio (SNR) of the measurements), the excitation (all vibrations of interest should be sufficiently excited (persistently excited)) and the length of the data record. Table 2 shows the SNR of each individual mode acceleration using different levels of measurement noise. $100 \mu \mathrm{g}$ measurement noise is referred to as a low SNR, $10 \mu \mathrm{g}$ as a medium high SNR, and $1 \mu \mathrm{g}$ as a high SNR.

Figures 5a, b show periodograms using measurement noise of $10 \mu \mathrm{g}$ and record lengths of 64 samples and 256 samples, respectively. Vertical lines in all PSD plots show the true frequencies. 

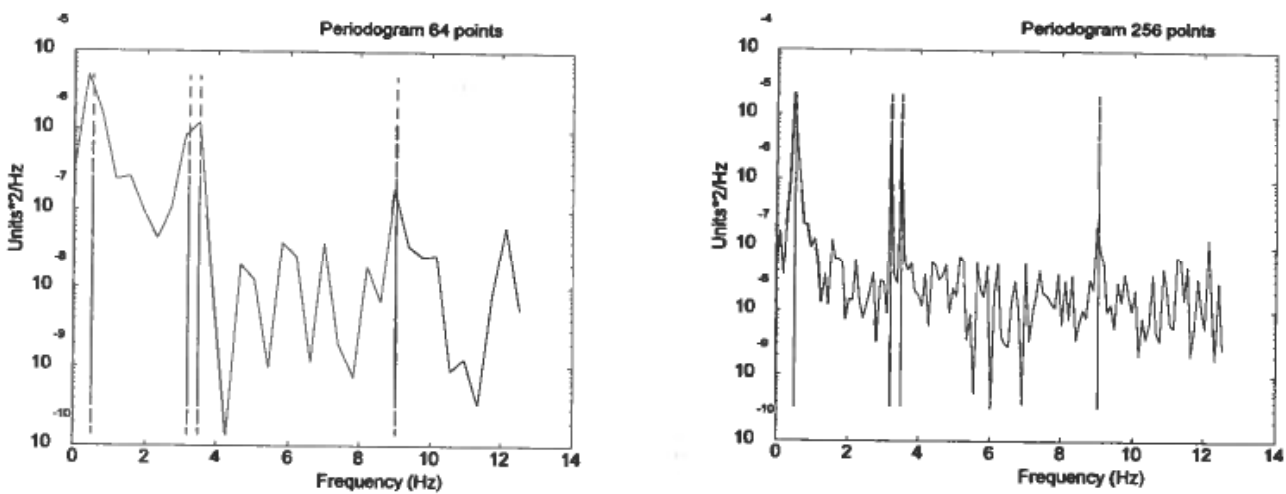

Figure 5a. Periodogram using 64 samples. Figure 5b. Periodogram using 256 samples.

Figure 5a,b. Periodogram using measurement noise of $10 \mu \mathrm{g}$ and record lengths of 64 samples and 256 samples, respectively.

Table 2. SNR of the individual accelerations for each individual vibration mode

\begin{tabular}{rcccc}
\hline \multicolumn{5}{c}{ SNR dB) } \\
\hline Noise & mode 1 & mode 2 & mode 3 & mode 4 \\
\hline $100 \mu \mathrm{g}$ & -2 & -11 & -12 & -19 \\
$10 \mu \mathrm{g}$ & 17 & 8 & 7 & 0 \\
$1 \mu \mathrm{g}$ & 37 & 28 & 26 & 20 \\
\hline
\end{tabular}

Figures $6 \mathrm{a}, \mathrm{b}$ show periodograms using measurement noise of $1 \mu \mathrm{g}$ and record lengths of 64 samples and 256 samples, respectively. Figures 7a, b show Welch SPD plots using measurement noise of $10 \mu \mathrm{g}$ and record lengths of 64 and 256 samples, respectively. The Welch PSD estimate uses a Hanning window on each segment with a segment length of 32 and 128 samples, respectively.

Figures $8 \mathrm{a}$, b show Welch SPD plots using measurement noise of $1 \mu \mathrm{g}$ and record
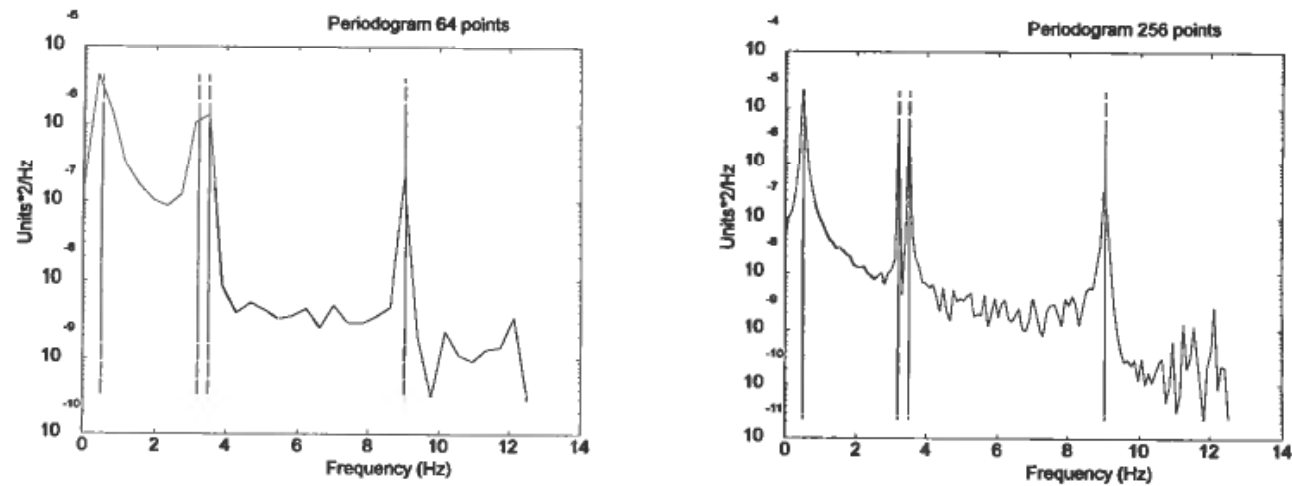

Figure 6a. Periodogram using 64 samples. Figure 6 b. Periodogram using 256 samples.

Figure 6a,b. Periodogram using measurement noise of $1 \mu \mathrm{g}$ and record lengths of 64 and 256 samples, respectively. 


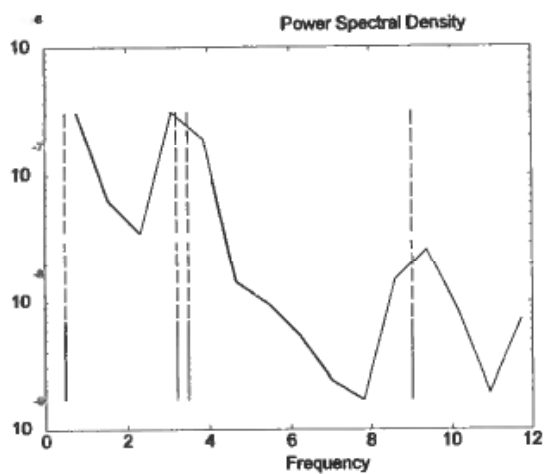

Figure 7a. Welch using 64 samples.

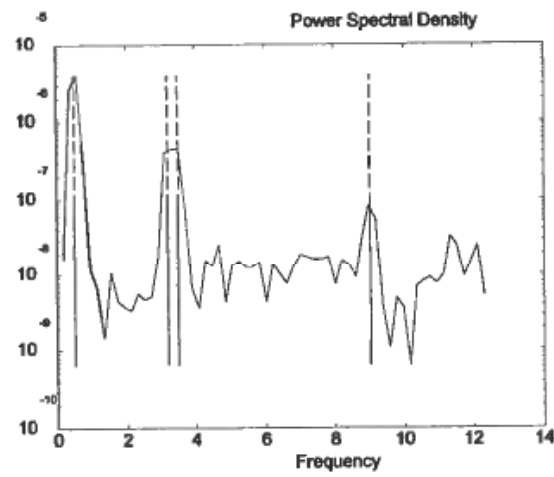

Figure $7 \mathrm{~b}$. Welch using 256 samples.

Figure 7a,b. Welch PSD plots using measurement noise of $10 \mu \mathrm{g}$ and record lengths of 64 and 256 samples, respectively.

lengths of 64 and 256 samples, respectively. The Welch PSD estimate uses a Hanning window on each segment with segment lengths of 32 and 128 samples, respectively.

\section{Comments to PSD results}

Figure 5a shows a periodogram using a record length of 64 samples and a measurement noise of $10 \mu \mathrm{g}$. It is not possible to separate the two narrow frequencies of modes 2 and 3, whereas the frequency estimate of mode 1 has a small frequency bias, and mode 4 is accurately estimated. Decreasing the record length worsens the frequency estimates. Increasing the record length to 256 samples, as shown in Figure 5b, improves the frequency estimates and all frequencies are accurately estimated. A record length of 128 samples improves the estimates compared to the 64 samples case, but fails to separate the two narrow frequencies of modes 2 and 3 . Reducing the measurement noise to $1 \mu \mathrm{g}$, as shown in Figure $6 \mathrm{a}$ and $\mathrm{b}$, does not significantly improve the frequency estimates compared to a measurement noise of $10 \mu \mathrm{g}$, but the fluctuations, shown in figure $5 \mathrm{~b}$, are reduced due to less measurement noise. Neither does a

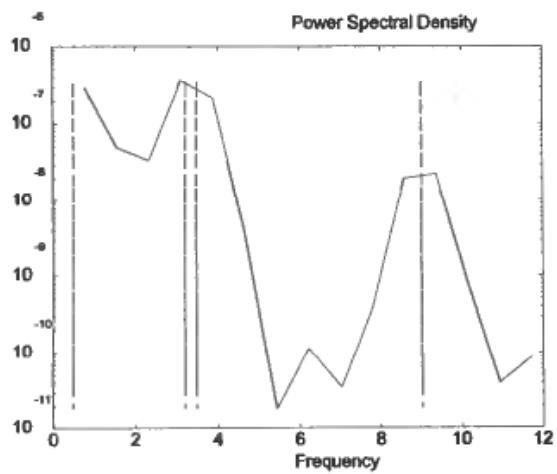

Figure $8 \mathrm{a}$. Welch using 64 samples.

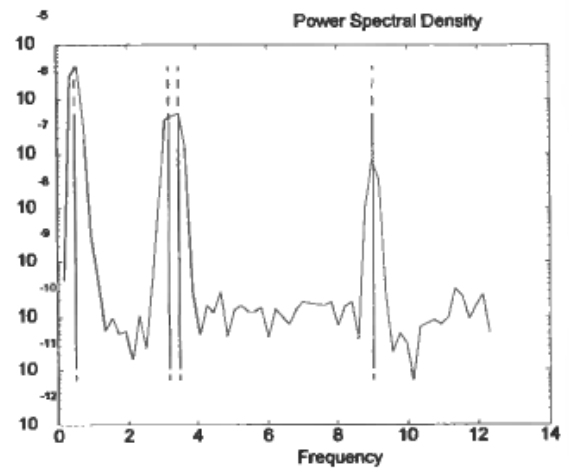

Figure 8b. Welch using 256 samples.

Figure 8a,b. Welch SPD plots using measurement noise of $1 \mu \mathrm{g}$ with record lengths of 64 and 256 samples. respectively. 


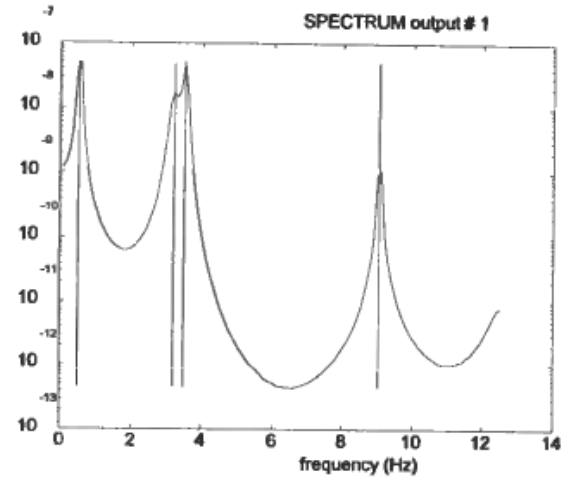

Figure 9a. AR(12) model.

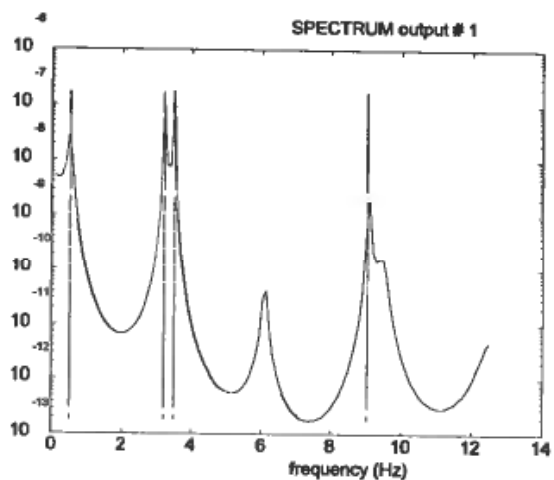

Figure $9 b$. AR(16) model.

Figure 9a,b. PSD plots calculated from parameter estimates using a forward-backward AR method. The measurement noise is $1 \mu \mathrm{g}$ and the record length is 30 samples.

further noise reduction make it possible to resolve mode 2 and 3 using a record length of 64 samples, corresponding to an observation time of 2.56 seconds.

The Welch PSD plots shown in Figures 7 and 8 give, compared to the periodogram, less accurate frequency estimates. Even a record length of 256 samples gives unsatisfactory separation of the two narrow frequencies of modes 2 and 3, whereas a record length of 512 samples gives satisfactory separation. The Welch plots show less noise than the periodogram, due to the windowing.

Increasing the measurement noise to $100 \mu \mathrm{g}$ gives too low SNR of the measurements to allow satisfactory frequency estimates using FFT methods, at least for a record length of up to 512 samples. Larger record lengths than 512 samples are assumed to require too long observation time and are not considered.

\section{Conclusion of the Nonparametric Methods}

Nonparametric methods such as the periodogram and the Welch spectral estimator are easy to use in estimating vibration frequencies in a space structure. However, all identification methods require the excitation signal to be persistently exciting, and the measurement noise to be of a suitable level. In our case $100 \mu \mathrm{g}$ measurement noise makes it impossible to separate the two narrowly spaced frequencies at $3.22 \mathrm{~Hz}$ and $3.50 \mathrm{~Hz}$, at least for record lengths up to 512 samples. Record lengths above 512 samples are not considered due to an observation time which is expected to be too long to be of practical interest. Reducing the measurement noise down to $10 \mu \mathrm{g}$ gives accurate frequency estimates for all frequencies with a record length of 256 samples.

Thus, a drawback of these methods is their need for long observation time. The above simulations show that a minimum observation time of $10 \cdot 24 \mathrm{sec}$. (256 samples) is required to separate the two narrow frequencies of modes 2 and 3, which are spaced $0.27 \mathrm{~Hz}$ apart. The simple periodogram performs best in the above simulations, but the periodogram is sensitive to noise and that may result in large noise fluctuations. Thus, filtering the data through a window as shown in the Welch method may be necessary in some cases. Windowing the data reduces the resolution, and consequently the periodogram has the best resolution of all FFT methods. 


\subsection{Parameter Identification using Parametric Methods}

These methods identify parameters which subsequently may be used to calculate vibration frequencies. Parametric methods give estimated parameters as output, but PSD plot will sometimes be used to give a quick overview of the frequency estimates.

\section{AR Methods}

Identification of parameters in AR models may be performed using some of the methods described in section 4 . Our space structure is modelled with four vibration frequencies and an AR model should at least contain eight parameters to identify all frequencies. Kay (1988) shows that the parameter accuracy may be improved by overparameterizing the model, i.e. specifying more parameters than the number of true parameters. How many redundant parameters it is advisable to use depends mainly on the measurement noise, the strength of the excitation signal, and the record length. Simulations (Skullestad 1995) show that more redundant parameters than twice the number of true parameters seldom give a significant increase in the parameter accuracy. Viberg (1993) recommends a record length of:

\section{Number of measurements $/ 3<$ Number of $A R$ parameters $<$ Number of measurements $/ 2$}

Thus, the record length may for AR identification be reduced significantly compared to the nonparametric methods. Simulations show that the record lengths may lie between 20 samples and 64 samples depending on the number of specified redundant parameters. Specifying 16 unknown parameters, i.e. eight redundant parameters, may require a longer data record length, due to more parameters to be determined, than specifying eight parameters. Even if accurate parameter estimates may be obtained with short data record lengths, a longer record length may sometimes improve the accuracy in noisy measurements, and may also move redundant poles away from the unit circle and thus, help separating true and false frequencies.

Table 3 tabulates estimated frequencies and their respective pole locations for an AR(8), AR(10), AR(12) and AR(16) model. The forward-backward method is used to estimate the parameters and the measurement noise is $1 \mu \mathrm{g}$. The record length is 30 samples.

Table 3. Location of estimated poles in z-domain (moduli and frequencies). [ ] shows redundant parameters. The record length is 30 samples

\begin{tabular}{|c|c|c|c|c|c|c|c|}
\hline \multicolumn{2}{|c|}{$\operatorname{AR}(8)$} & \multicolumn{2}{|c|}{$\mathrm{AR}(10)$} & \multicolumn{2}{|c|}{$\mathrm{AR}(12)$} & \multicolumn{2}{|c|}{$\mathrm{AR}(16)$} \\
\hline Modul. & Freq. $\mathrm{Hz}$ & Modul. & Freq. $\mathrm{Hz}$ & Modul. & Freq. $\mathrm{Hz}$ & Modul. & Freq. $\mathrm{Hz}$ \\
\hline 0.964 & 0.57 & 0.996 & 0.56 & 0.999 & $0 \cdot 54$ & 1.012 & $0 \cdot 52$ \\
\hline \multicolumn{2}{|c|}{2 real poles } & 0.954 & $3 \cdot 25$ & 0.969 & $3 \cdot 17$ & 0.996 & $3 \cdot 23$ \\
\hline 0.992 & 3.43 & 0.962 & 3.60 & 0.987 & $3 \cdot 54$ & 0.998 & $3 \cdot 50$ \\
\hline \multirow[t]{3}{*}{0.998} & 9.04 & 0.999 & 9.04 & 1.002 & 9.03 & 1.002 & $9 \cdot 02$ \\
\hline & & {$[0 \cdot 722$} & 11.96] & {$[0.702$} & 8.96] & {$[0 \cdot 988$} & 6.19] \\
\hline & & & & [2 re: & ${ }^{2} \mathrm{p}$ & \multicolumn{2}{|c|}{ [4 real poles] } \\
\hline
\end{tabular}

The moduli show how close to the unit circle the poles of the AR model were estimated. Figures 9a, b show PSD plots calculated from the parameter estimates of the $\mathrm{AR}(12)$ and AR(16) model shown in Table 3. 


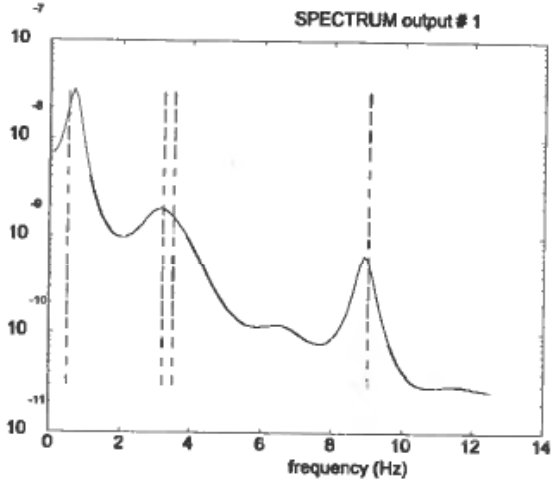

Figure 10a. AR(12) model.

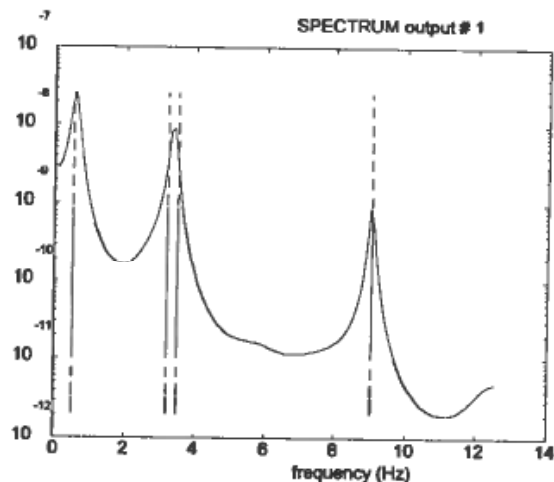

Figure 10b. AR(16) model.

Figure 10a,b. PSD plots calculated from parameter estimates using an autocorrelation AR method. The measurement noise is $1 \mu \mathrm{g}$ and the record length is 30 samples.

Figures 10a, b show PSD plots calculated from parameter estimates using an autocorrelation AR method.

Figures 11a, b show PSD plots calculated from parameter estimates using a covariance AR method.

\section{Comments to AR Methods}

Table 3 shows that the AR(8) model identifies mode 1 to $0.57 \mathrm{~Hz}$, mode 3 to $3.43 \mathrm{~Hz}$ and mode 4 to $9.04 \mathrm{~Hz}$. Mode 2 is not found, but instead two real frequencies are estimated. The AR(10) model improves the parameter accuracy, but the two redundant poles form a false frequency at $11.96 \mathrm{~Hz}$. However, the poles of the false frequency are located far from the unit circle, modulus $=0.722$, and are easy to separate from the true frequencies which have moduli $>0.95$. The $A R(12)$ model gives more accurate frequency estimates than the AR(10) model and the AR(16) model is slightly more accurate than the AR(12) model. No improvements are made for model orders above 16. The AR(12) model has 4 redundant poles, two are estimated to be real and two are

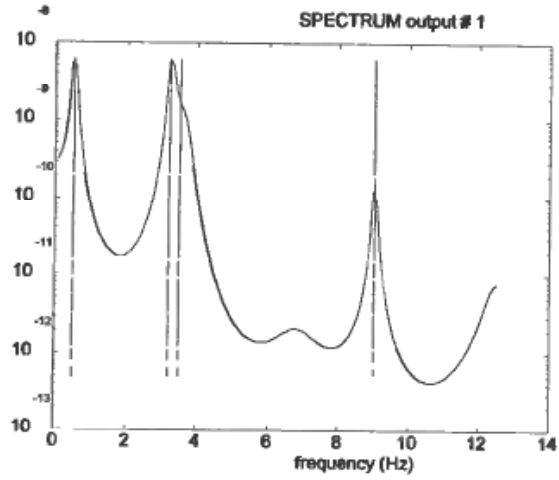

Figure 11a. AR(12) model.

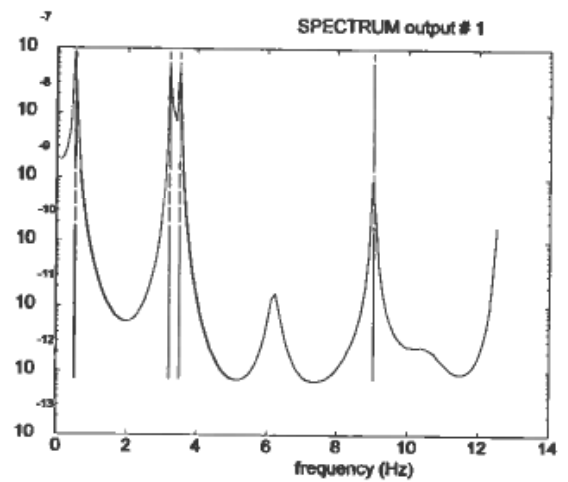

Figure 11b. AR(16) model.

Figure 1 la,b. PSD plots calculated from parameter estimates using a covariance AR method. The measurement noise is $1 \mu \mathrm{g}$ and the record length is 30 samples. 


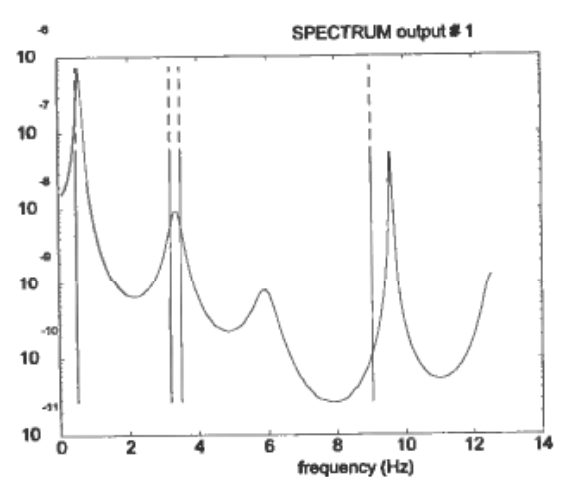

Figure 12a. AR(12) model.

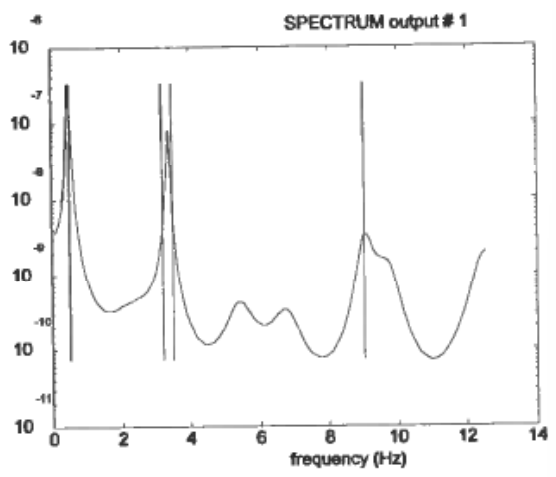

Figure 12b. AR(16) model.

Figure 12a,b. PSD plots calculated from parameter estimates using a forward-backward AR method. The measurement noise is $10 \mu \mathrm{g}$ and the record length is 30 samples.

complex, but located far from the unit circle, figure 9a. The AR(16) model estimates four false poles close to the unit circle, easily seen in figure $9 \mathrm{~b}$, as frequency peaks at $6.19 \mathrm{~Hz}$ and $9.47 \mathrm{~Hz}$. Increasing the number of redundant parameters increases the chances of estimating false frequencies close to the unit circle. If redundant complex poles are located close to the unit circle, it may be difficult to separate false and true vibration frequencies unless one knows the number of frequencies or their approximate location.

It is possible to reduce the number of samples down to approximately 20 samples for the AR(8), AR(10) and AR(12) models without losing too much accuracy. The AR(16) model requires more than 30 samples to determine the 16 unknown parameters accurately. Increasing the number of samples may slightly improve the parameter estimates for low SNR measurements, but not significantly. Normally, an AR(8) model has too few parameters to resolve the narrow spaced frequencies of mode 2 and 3 , but in some low-noise measurement situations eight parameters may be sufficient.

Results from the autocorrelation method shown in figure $10 \mathrm{a}$ and $\mathrm{b}$ are less accurate than the results from the forward-backward method. The experiments show that the autocorrelation method is not suitable for estimating poles close to the unit circle, which is the situation when vibration frequencies in low-damped space structures are estimated.

Results from the covariance method shown in figures 11a and $b$ reveal approximately the same parameter accuracy as the forward-backward method or slightly worse. Thus, the covariance method may also be used to estimate poles close to the unit circle.

From the above simulations the forward-backward method seems to give the best parameter accuracy. All simulations so far have made use of a measurement noise of $1 \mu \mathrm{g}$, because AR methods are most suitable for high SNR measurements and in some cases medium high SNR measurements. A measurement noise of $10 \mu \mathrm{g}$, referred to as a medium high SNR measurement signal, identifies mode 1 and 4 , but is not able to separate the two narrow frequencies mode 2 and 3 . The acceleration due to mode 4 , Table 1, has the lowest amplitude, but is often accurately identified. This is due to the fact that AR methods pay great attention to the high frequencies in a measurement while low frequencies may be more difficult to identify accurately (Ljung 1987). 

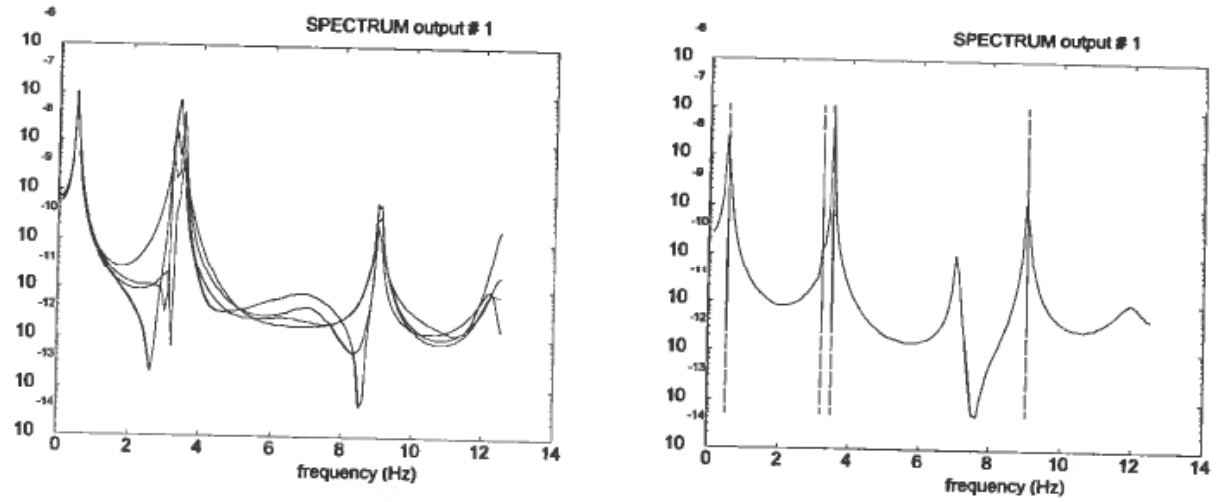

Figure 13. Different number of $\mathrm{C}$ parameters.

Figure 14. ARMA(12,6).

The results shown in Figures 12a, b refer to the same experiment as those in Figures $9 \mathrm{a}, \mathrm{b}$ except for the fact that the measurement noise has now been increased to $10 \mu \mathrm{g}$.

\section{ARX Methods}

ARX models give approximately the same parameter accuracy as AR models and are not shown (Skullestad 1995).

\section{Methods}

Simulations documented in this paper are limited to Gaussian distributed white noise and in that case IV methods do not give better results than AR and ARX methods (Skullestad 1995).

\section{ARMA and ARMAX Methods}

So far chapter 5 has described identification of vibration frequencies using AR, ARX and IV models and associated methods. These models/methods work well for short data records and mainly high SNR measurements but, unfortunately, they become less accurate for low SNR measurements and frequently also for medium high SNR measurements. In most AR and ARX identification models the noise is more or less ignored, this also applies to the models used in this paper. In contrast to these methods, ARMA and ARMAX models all have the feature of allowing a model of the noise. If the chosen noise model contributes to a more accurate mathematical model of the process than no noise model at all, then one may expect more accurate parameter estimates from ARMA and ARMAX models.

Parameter estimates from ARMA and ARMAX models may be identified using analytical methods or numerical search methods. This paper is limited to ARMA and ARMAX methods using a Gauss-Newton numerical search method.

An ARMA model requires a specification of both the A- and C-polynomials, i.e. the number of both A- and C-parameters have to be specified (ARMA(A,C)). Experiments show that the parameter accuracy is relatively insensitive to the complexity of the noise model as long as the number of C-parameters is $\geq 2$. The presumably correct model of the form ARMA $(8,7)$ or ARMA $(8,8)$ gives approximately the same frequency as an $\operatorname{ARMA}(8,6)$ model. The results from ARMA models using $6 \mathrm{C}$-parameters are therefore chosen for further study. 


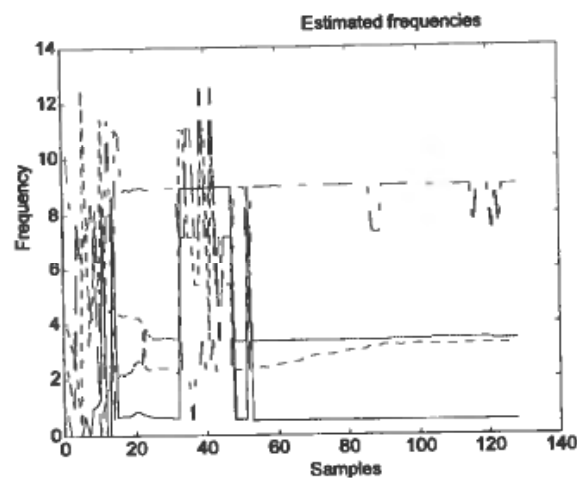

Figure 15. AR(16) model using RLS with forgetting factor.

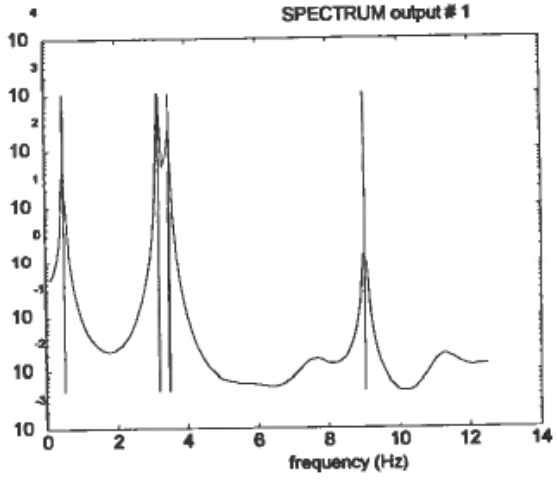

Figure 16. ARMA(16,6) and RPLR.

Figure 13 shows PSD plots using an A-polynom of degree 12 and different degrees of the C-polynom. The record length is 128 samples and the measurement noise is $1 \mu \mathrm{g}$.

Additionally, an ARMA model gives increasing parameter accuracy when the number of parameters in the A-polynom is increased, see Table 4 . Table 4 tabulates estimated frequencies and their respective pole locations for $\operatorname{ARMA}(8,6)$, $\operatorname{ARMA}(10,6), \operatorname{ARMA}(12,6)$ and ARMA $(16,6)$ models. The measurement noise is $1 \mu \mathrm{g}$ and the record length is 128 samples. Table 4 should be compared with Table 3 . To produce accurate parameter estimates an ARMA model requires a longer record length than an AR model, and a record length of 128 samples may be a good choice. A record length of 64 samples gives slightly less accurate estimates, but may be used.

Table 4. Location of estimated poles in $\mathbf{z}$-domain (moduli and frequencies). [] shows redundant parameters. The record length is 128 samples

\begin{tabular}{|c|c|c|c|c|c|c|c|}
\hline \multicolumn{2}{|c|}{$\operatorname{ARMA}(8,6)$} & \multicolumn{2}{|c|}{$\operatorname{ARMA}(10,6)$} & \multicolumn{2}{|c|}{$\operatorname{ARMA}(12,6)$} & \multicolumn{2}{|c|}{$\operatorname{ARMA}(16,6)$} \\
\hline Modul. & Freq. $\mathrm{Hz}$ & Modul. & Freq. $\mathrm{Hz}$ & Modul. & Freq. $\mathrm{Hz}$ & Modul. & Freq. $\mathrm{Hz}$ \\
\hline 0.997 & 0.51 & 0.999 & 0.51 & 0.999 & 0.51 & 0.999 & 0.51 \\
\hline 0.667 & $11 \cdot 26$ & 0.998 & $3 \cdot 21$ & 0.997 & $3 \cdot 22$ & 0.998 & $3 \cdot 22$ \\
\hline 1.005 & 3.37 & 0.996 & $3 \cdot 50$ & 0.998 & $3 \cdot 51$ & 0.999 & 3.50 \\
\hline \multirow[t]{4}{*}{0.998} & $9 \cdot 02$ & 0.997 & 9.02 & 0.995 & $9 \cdot 02$ & 0.997 & $9 \cdot 02$ \\
\hline & & \multicolumn{2}{|c|}{ [2 real poles] } & {$[1.008$} & 7-06] & {$[0.929$} & 5-81] \\
\hline & & & & \multicolumn{2}{|c|}{ [2 real poles] } & {$[0.900$} & $\begin{array}{r}7 \cdot 85] \\
10 \cdot 81]\end{array}$ \\
\hline & & & & & & \multicolumn{2}{|c|}{ [2 real poles] } \\
\hline
\end{tabular}

\section{Comments to ARMA models}

Table 4 shows that the ARMA method gives more accurate parameter estimates than the AR method shown in Table 3 . Especially the low order ARMA $(10,6)$ model gives more accurate parameter estimates than the comparable AR(10) model. An ARMA model of the form ARMA $(8,7)$ or $\operatorname{ARMA}(8,8)$ is not able to separate modes 2 and 3 and overparameterizing of the A-polynom is required in order to separate these modes. The ARMA $(12,6)$ model estimates one complex redundant pole pair outside the unit circle, which may make it very difficult to differentiate the resulting frequency at 

7.06 $\mathrm{Hz}$ from true frequencies. A PSD plot of the $\operatorname{ARMA}(12,6)$ model is shown in figure
14.

Figure 14 does not indicate a true frequency at $3.22 \mathrm{~Hz}$, i.e. mode 2 seems to be lost, but the explanation is that the $\mathrm{C}$ polynom forms a complex zero at the same location as the complex pole of mode 2 and, thus, cancels the frequency of mode 2.

Table 4 makes use of a measurement noise of $1 \mu \mathrm{g}$. Increasing the measurement noise to $10 \mu \mathrm{g}$ gives less accurate estimates. Modes 1 and 4 are still accurately estimated, but the narrow frequencies of modes 2 and 3 are never properly separated.

Numerical search methods make use of initial conditions, and if no information about the parameters are given these values are taken as zero. Thus, if we from physical knowledge or from experiments have better knowledge of the parameters than assuming them to be zero, these values may be taken as initial values. Simulations have shown that the estimation time is shorter with reasonable a priori information (initial conditions), but this does not always give better parameter estimates. High SNR measurements give accurate parameter estimates independent of initial conditions. Medium high SNR measurements may give parameter improvements if a priori values are used. Low SNR measurements, in most cases, give only marginal improvements, if any at all. Simulations with our spacecraft model using a measurement noise of $1 \mu \mathrm{g}$ give accurate parameter estimates which turn out to be independent of the initial conditions. $10 \mu \mathrm{g}$ measurement noise is a more difficult identification task and only small parameter improvements are achieved with very accurate initial conditions; the SNR is too low even for this numerical search method.

\section{ARMAX Model}

An ARMAX model requires parameterizing of the B-polynom and a process delay in addition to the ARMA model. Simulations have shown (Skullestad 1995) that there is no benefit in using an ARMAX model instead of an ARMA model. These two models give approximately the same parameter accuracy, but the ARMAX model is slower due to a higher number of parameters and, hence, often needs a longer record length.

\section{Recursive Methods}

The AR, ARX, ARMA and ARMAX models can all be handled by recursive algorithms. MATLAB's System Identification Toolbox contains different methods for estimating parameters from the above models. This paper will look at a recursive least square method with forgetting factor (RLS) and a recursive pseudolinear regression method (RPLR). The recursive ARX (RARX) routine contained in this toolbox handles AR and ARX models, whereas the RPLR routine handles ARMA and ARMAX models as well as AR and ARX models.

Figure 15 shows the estimated frequencies versus the number of samples (number of iterations) for an AR(16) model using an RLS method with forgetting factor. The forgetting factor is 0.98 . The initial covariance matrix is $1000000^{*} \mathrm{I}$. Measurement noise is $10 \mu \mathrm{g}$.

Table 5 shows true frequencies estimated from an AR(12) and AR(16) model using an RPL method with forgetting factor 0.98 , and the initial covariance matrix is $1000000^{*} \mathrm{I}$. The estimated frequencies are collected after 64 iterations and 256 iterations respectively.

Table 6 shows true frequencies estimated from an $\operatorname{ARMA}(12,6)$ and $\operatorname{ARMA}(16,6)$ 
Table 5. Estimated true frequencies from AR models collected after 64 and 256 iterations using an RLS method with forgetting factor $=0 \cdot 98$. Measurement noise is $1 \mu \mathrm{g}$

\begin{tabular}{lccccc}
\hline & \multicolumn{3}{c}{ RLS method with forgetting factor } \\
\cline { 2 - 3 } & \multicolumn{2}{c}{64 iterations } & & \multicolumn{2}{c}{256 iterations } \\
\cline { 2 - 3 } \cline { 5 - 5 } Frequency $(\mathrm{Hz})$ & $\mathrm{AR}(12)$ & $\mathrm{AR}(16)$ & & $\mathrm{AR}(12)$ & $\mathrm{AR}(16)$ \\
\hline Mode 1 & 0.55 & 0.55 & & 0.51 & 0.51 \\
Mode 2 & 3.36 & 2.94 & & 3.32 & 3.20 \\
Mode 3 & 4.08 & 3.37 & & 3.63 & 3.50 \\
Mode 4 & 9.00 & 9.00 & & 9.02 & 9.02 \\
\hline
\end{tabular}

Table 6. Estimated true frequencies from ARMA models collected after 64 and 256 iterations using an RPLR method. Measurement noise is $1 \mu \mathrm{g}$

\begin{tabular}{lccccc}
\hline & \multicolumn{4}{c}{ RPLR method } \\
\cline { 2 - 3 } \cline { 5 - 6 } Frequency (Hz) & ARMA(12,6) & ARMA(16,6) & & ARMA(12,6) & ARMA (16,6) \\
\cline { 2 - 3 } & 0.55 & 0.55 & & 0.51 & 0.51 \\
Mode 1 & 3.36 & 2.94 & & 3.32 & 3.20 \\
Mode 2 & 4.08 & 3.37 & & 3.63 & 3.50 \\
Mode 3 & 9.00 & 9.00 & & 9.02 & 9.02 \\
Mode 4 & & &
\end{tabular}

model using an RPLR method, with an initial covariance matrix of $1000000^{*} \mathrm{I}$. The estimated frequencies are collected after 64 iterations and 256 iterations respectively.

\section{Comments to Recursive Methods}

Recursive methods are less effective than batch methods, but the parameter accuracy increases with an increasing number of iterations and after 256 iterations both the AR and ARMA models, using an RPL and RPLR method respectively, become quite accurate. The redundant poles seem to locate themselves at a distance away from the unit circle. Figure 16 shows a PSD plot of the ARMA(16,6) model after 256 iterations. This figure shows that the false frequencies do not have sharp peaks, i.e. the estimated poles are relatively far from the unit circle.

\section{Conclusion of the Parametric Methods}

Low-damped space structures have poles close to the unit circle in the z-domain and it is important to choose methods suitable for that pole location. The forward-backward and covariance methods can be recommended using AR models. AR methods require very short record lengths and the computation cost is low. The accuracy is good for high SNR measurements, but unfortunately the parameter accuracy decreases with decreasing SNR.

ARX methods give approximately the same parameter accuracy as AR methods. The record length may be increased due to more parameters and hence also the computation cost is higher.

IV methods do not give improvements compared to ARX methods due to the assumption of white measurement noise.

The parameters of the ARMA model are estimated using a Gauss-Newton numerical 
search method. The ARMA method gives slightly better parameter estimates than the $\mathrm{AR}$ and ARX methods, but the computation cost is, due to the numerical search routine, much higher.

ARMAX methods give approximately the same accuracy as ARMA methods.

Recursive methods give less accurate parameter estimates than the respective batch methods, but by allowing enough iterations it may be possible to achieve acceptable accuracy.

\section{Conclusion}

Analytical parametric methods are faster than nonparametric methods and may give better vibration frequency estimates for high SNR measurements. Medium high SNR measurements may require a numerical search algorithm to give the best vibration frequency estimates. All parametric methods, during the work reported here, have problems in accurately identifying vibration frequencies using low SNR measurements. Nonparametric methods may give better results than parametric methods for low SNR measurements, but at the cost of very long observation times.

\section{Acknowledgements}

This research has been supported by the Royal Norwegian Council for Scientific and Industrial Research (NTNF).

\section{REFERENCES}

BøE, S. (1991). Lecture notes in signal analysis. IN 357, University of Oslo.

Egeland, O., KaneSTRøM, R., and OMA, P. N. (1991). Modellering og simulering av fleksible romfartøyer. Technical Report no. 91-113, Norwegian Institute of Technology (NTH).

Golub, G. H., and vaN LOAN, C. F. (1989). Matrix Computations. (Johns Hopkins University Press).

Hallingstad, O. (1976). Maximum likelihood identifikasjon av parametre $\mathrm{i}$ ulineære tilstandrommodeller. PhD Thesis, Norwegian Institute of Technology (NTH).

IRGENS, F. (1990). Dynamikk. (Tapir, Norway).

Johansson, R. (1993). System Modeling \& Identification. (Prentice Hall, New York).

JoSHI, S. M. (1989). Control of Large Flexible Space Structures. (Springer-Verlag, New York).

KAY, S. M. (1988). Modern Spectral Estimation. (Prentice Hall, New York).

DE LAFOnTAine, J. (1990). Flexible Mode Dynamic Equations, part A and B, hand written memorandum.

LANDAU, I. D. (1990). System Identification and Control Design. (Prentice Hall, New York).

LJUNG, L. (1987). System Identification, Theory for the User. (Prentice Hall, New York).

LJUNG, L. and SÖDERSTRÖM, T. (1983). Theory and Practice of Recursive Identification. (The MIT Press).

MarPle, S. L. (1987). Digital Spectral Analysis with Applications. (Prentice Hall, New York).

RAO, S. (1990). Mechanical Vibrations. (Addison Wesley).

Skullestad, A. A. (1995). Identification of Vibration Parameters in a Space Structure. PhD Thesis, University of Oslo.

SöDERSTRÖM, T. and STOICA, P. (1989). System Identification. (Prentice Hall, New York).

THERRIEN, C. W. (1992). Discrete Random Signals and Statistical Signal Processing. (Prentice Hall, New York).

VIBERG, M. (1993). Hand written memorandum. 